\title{
Shortcuts in Cosmological Branes
}

\author{
Elcio Abdalla, Adenauer G. Casali and Bertha Cuadros-Melgar \\ Instituto de Fisica, Universidade de São Paulo \\ C.P.66.318, CEP 05315-970, São Paulo, Brazil.
}

\begin{abstract}
We aim at gathering information from gravitational interaction in the Universe, at energies where quantum gravity is required. In such a setup a dynamical membrane world in a space-time with scalar bulk matter described by domain walls, as well as a dynamical membrane world in empty Anti de Sitter space-time are analysed.

We later investigate the possibility of having shortcuts for gravitons leaving the membrane and returning subsequently. In comparison with photons following a geodesic inside the brane, we verify that shortcuts exist. For late time universes they are small, but for some primordial universes they can be quite effective.

In the case of matter branes, we argue that at times just before nucleosynthesis the effect is sufficiently large to provide corrections to the inflationary scenario, especially as concerning the horizon problem and the Cosmological Background Radiation.
\end{abstract}

\section{Introduction}

Although the Standard Model of particle physics has been established as the uncontested theory of all interactions down to distances of $10^{-17} \mathrm{~m}$, there are good reasons to believe that there is a new physics arising soon at the experimental level [1]. On the other hand, string theory provides an excelent background to solve long standing problems of theoretical high energy physics. It is by now a widespread idea that M-theory [2] can be a reasonable description of our Universe. In the field theory limit, it is described by a solution of the (eventually 11-dimensional) Einstein equations with a cosmological constant by means of a four dimensional membrane. In this 
picture only gravity survives in the extra dimensions, while the remaining matter and gauge interactions are typically four dimensional.

This avenue presents us a possibility of tackling with two different problems at the same time, namely a means of testing the up to now far from experimental and observational data string theory, and a theoretical framework to cosmology, whose theoretical background needs a full understanding of quantum gravity in order to correctly deal with the puzzling question of the initial singularity.

General Relativity always evaded quantization. Einstein gravity is a nonrenormalizable theory at a low loop level [3], and atempts to include supersymmetry in order to cancel divergencies only postponed the problem to a higher loop [4]. String Theory showed a way from its very basis of how to succesfully quantize gravity. At the same time, and especially after the discovery of the anomaly cancellation mechanism [5], now even sometimes quoted as the first superstring revolution, strings are taken as quasi unique in their description. Moreover, with the discovery of the duality symmetry such a uniqueness has been further enhanced [6], leading to the concept of a universal theory, the so called M-theory largely unknown, describing an eleven dimensional misterious, master theory, mother of all string descriptions on their side related by duality and containing in their field theory limit a version of 11-dimensional supergravity [7].

The key question however lies on what can turn a higher dimensional theory into something realistic, describing our four dimensional world. Some hints are known since almost a century and have been used before in the context of supergravity. It is the Kaluza Klein approach [8], where extra dimensions are compactified being curled up in such a size that they cannot be observed in the daily life, that in spite of being appealing, has encountered several problems being still an active field of inquire. Still there are proposals where some of these dimensions are large enough to be probed by microphysics [9].

Recently, Horava and Witten [10] considered M-theory in a rather intriguing situation. It has been argued that our universe can be seen as a solution of a higher dimensional theory. Strings can be open or closed. The closed string sector contains gravity and further components. The open string sector contains matter fields isolated into their extremities. In this proposed picture the open string extremities containing the matter fields are restrained to live in the physical universe, which in Horava and Witten's proposal is described 
by a membrane.

Later on, Randall and Sundrum [36] described the universe as a solution of higher dimensional field equations with boundary conditions describing the membrane. As a result, they obtained a space with full fledged extra dimensions but with a warp such that the effective penetration of gravitons into the extra dimensions is small, and the effective gravitational interaction is observationally four dimensional. In this picture there is a possibility that gravitational fields, while propagating out of the brane, speed up reaching farther distances in smaller time as compared to light propagating inside the brane, a scenario that for a resident of the brane implies shortcuts [11] — [15].

It is our aim in this work to further develop these ideas in the case of a FRW brane Universe [16]. The subject was developed until now from the point of view of the brane [17], where all time dependence is embedded in the bulk metric written in gaussian coordinates. The price paid is the complicated form of the bulk metric and, consequently, the complicated behaviour of geodesics in the bulk. However, if we treat the problem from the point of view of the bulk, where the brane evolves in a non-trivial way in a static AdS background, we can construct explicitly the causal structure of null geodesics leaving and subsequently returning to the brane. As it turns out, shortcuts are common, although harmless at the present days (the delay is vanishingly small), but could be large in the era before nucleosynthesis.

Moreover, one of the main goals of string theory nowadays is to prove itself able of coping with experimental evidences. Branes have been shown to be useful tools to understand the physics of strings and M-theory [18]. As it has also recently been pointed out [19], brane Universes, such as the one described above, could imply the existence of relics of the extra dimensions in the cosmic microwave background. Unfortunately, recent developments with inflation guided by a scalar field on the brane indicate that the consistency equation is preserved [20]. In this work however, we show that if inflation took part on the brane, the causal structure is definitely changed by those gravitational shortcuts, possibly leading to a non-usual period of causal evolution of scales. This could be responsible for distinct predictions in the cosmic microwave background structure for inflationary models.

This scenario has been proposed as an actually realizable possibility [21] [27]. In [23] it has been shown that in some scenarios shortcuts are very difficult to be detected today because of the extremely short delay of the photon as compared to the graviton coming from the same source. 


\section{Further Motivations and Brane Cosmology}

The Planck mass is the natural scale where string effects become important. However, it is not possible to achieve such an energy level in particle physics accelerators. Nonetheless, as we mentioned above, cosmology may provide an alternative laboratory for string theory. From the eighties several authors tried to analysed the kind of cosmology arising from string inspired models, which are essentially general relativity in higher dimensions together with scalar and tensor fields. In case we also introduce the brane concept, a consistent picture of the brane universe is achieved, and we can describe the evolution of the universe by means of solutions of the Einstein field equations in higher dimensions with a four dimensional membrane.

Extensive use of specific properties of strings, such as the T-duality, may provide simple explanations of several properties of the universe, though of course an observational check is missing. Nonetheless, a pre big-bang scenario can be described providing a very sophisticated physical picture of the universe. Several solutions also indicating inflation have been obtained in what is now usually called brane cosmology.

It is thus essential to provide means of comparing those results to some observational data, which is the largest difficulty in string theory. The best proposal is to use the extremely sophisticated data of the Cosmic Microwave Background, first obtained from the pioneering COBE satelite and more recently by the detailed analysis of the WMAP. Such observations are opening the avenue for precision cosmology, and results with error bars less a percent are now available. In such a case one can compare the predictions of inflationary models with observations, and even quantitatively distinguish between different inflationary scenarios with a chance of favouring or discarding string inspired models of cosmology or brane cosmology.

The definition of a scalar potential directly from string theory has been an important issue. In [24] an attempt was made in order to derive the scalar interaction from a brane-brane interaction. This kind of approach reviewed in [25] has been very recently taken over in [26]. A few different and new forms for the inflaton potential have been derived, showing that at least one can hope to derive inflationary models directly from the properties which caracterize string theory and its consequences, namely M-theory and branes. 


\section{The junction conditions}

The formulation of proper junction conditions at surfaces of discontinuity is a fundamental problem in gravitational theory. Well-known examples are the Schwarzschild and Oppenheimer-Snyder problems, which require the junction of the interior field of a static or collapsing star to the exterior vacuum field.

In newtonian theory the problem is directly solved by imposing the standard continuity and jump conditions connecting the potential and its first derivatives across the surface. In general relativity, however, the gravitational potential is not only determined by the smoothness of physical conditions but also by the smoothness of the coordinates we are using to describe spacetime.

A pioneering work on these subjects was made in 1922 by C. Lanczos [28], on which W. Israel [29] based his landmark formalism forty years later. Independent of these works, G. Darmois [30] derived junction conditions for the special case of a boundary surface, i.e. a surface through which both the metric and the extrinsic curvature tensor are continuous. All these ideas are now known as the Darmois-Israel junction/thin-shell formalism, which has found wide application in general relativity and cosmology, including further studies of gravitational collapse, the evolution of bubbles and domain walls in a cosmological setting, wormholes and more recently in brane cosmology.

In this section we review the junction formalism as it was first derived by Israel. We also derive the Darmois-Israel conditions from the gravitational action in the context of $D$-dimensional theories and give an alternative approach using distribution theory.

\subsection{The Formalism}

Consider a spacetime $M$ (pseudo-riemannian manifold with signature $(-+$ $++)$ ) with metric $g_{\alpha \beta}\left(x^{\gamma}\right)$ in the coordinate system $\left(x^{\gamma}\right)$. The absolute derivative of a smooth vector function $\mathbf{A}$ defined on a curve on this spacetime parametrized by $t$ is given by

$$
\nabla_{t} A^{\alpha} \equiv \frac{\partial A^{\alpha}}{\partial t}+A^{\lambda} \Gamma_{\lambda \mu}^{\alpha} \frac{d x^{\mu}}{d t}
$$

Let $\Sigma$ be a smooth hypersurface in $M$ with metric $g_{i j}\left(\xi^{c}\right)$ in the coordinates $\xi^{c}$, which separates $M$ into two four-dimensional manifolds $M^{-}$and $M^{+}$, each containing $\Sigma$ as part of its boundary. 
The unit 4-normal $\mathbf{n}$ to this hypersurface in $M$ labels $\Sigma$ as timelike (spacelike) for $\epsilon(\mathbf{n}) \equiv \mathbf{n} \cdot \mathbf{n}=-1$ (1).

We define a natural frame of three linearly independent tangent vectors $e_{(i)}^{\alpha}$ associated with the intrinsic coordinates $\xi^{i}$ as

$$
e_{(i)}^{\alpha}=\frac{\partial x^{\alpha}}{\partial \xi^{i}},
$$

which gives the induced metric on $\Sigma$ as

$$
g_{i j} \equiv \mathbf{e}_{(i)} \cdot \mathbf{e}_{(j)}=\frac{\partial x^{\alpha}}{\partial \xi^{i}} \frac{\partial x^{\beta}}{\partial \xi^{j}} g_{\alpha \beta} .
$$

The intrinsic covariant derivative of $\mathbf{A}$ with respect to $\xi^{i}$ is the projection of the vector $\partial \mathbf{A} / \partial \xi^{j}$ onto $\Sigma$,

$$
A_{i ; j}=\mathbf{e}_{(i)} \cdot \frac{\partial \mathbf{A}}{\partial \xi^{j}}=\frac{\partial A}{\partial \xi^{j}}-A_{h} \Gamma_{i j}^{h} .
$$

We see that intrinsic covariant differentiation does not depend on the nature of the embedding. Properties of a non-intrinsic character enter when we consider the way in which $\Sigma$ "bends" in $M$. This is measured by the variations $\partial \mathbf{n} / \partial \xi^{i}$ of the unit normal,

$$
\partial \mathbf{n} / \partial \xi^{i}=K_{i}^{j} \mathbf{e}_{(j)},
$$

what defines the extrinsic curvature 3-tensor $K_{i j}$ of $\Sigma$,

$$
K_{i j}=e_{(j)} \cdot \frac{\partial \mathbf{n}}{\partial \xi^{i}} \equiv \frac{\partial x^{\alpha}}{\partial \xi^{i}} \frac{\partial x^{\beta}}{\partial \xi^{j}} \nabla_{\alpha} n_{\beta}=-n_{\gamma}\left(\frac{\partial^{2} x^{\gamma}}{\partial \xi^{i} \partial \xi^{j}}+\Gamma_{\alpha \beta}^{\gamma} \frac{\partial x^{\alpha}}{\partial \xi^{i}} \frac{\partial x^{\beta}}{\partial \xi^{j}}\right) .
$$

Let $K_{i j}^{-}, K_{i j}^{+}$be the extrinsic curvatures of $\Sigma$ associated with its embeddings in $M^{-}, M^{+}$. If $K_{i j}^{-} \neq K_{i j}^{+}, \Sigma$ is called a singular hypersurface of order one, surface layer or thin shell. If $K_{i j}^{-}=K_{i j}^{+}, \Sigma$ is called a hypersurface of higher order or boundary surface.

The Darmois conditions for the joining of $M^{-}$and $M^{+}$through $\Sigma$ are [30]

$$
\begin{aligned}
{\left[g_{i j}\right] } & =0, \\
{\left[K_{i j}\right] } & =0,
\end{aligned}
$$


where $[X]=X^{+}-X^{-}$is the jump of $X$ through the hypersurface $\Sigma$.

A boundary surface satisfies both equations, while a thin shell only satisfies (7). Condition (8) as it stands is ambiguous since the orientation of the 4 -vector field $\mathbf{n}$ has not been specified. The Israel formalism will require the normals in $M$ to point from $M^{-}$to $M^{+}$. We should stress that the majority of the existing literature deals with spherical symmetry where the direction of the normal is clear, but in more complicated cases great care must be taken.

The Israel formulation [29] of thin shells follows from the Lanczos equation [28]

$$
\left[K_{i j}\right]-g_{i j}\left[K_{a}^{a}\right]=-8 \pi G S_{i j},
$$

or equivalently

$$
\left[K_{i j}\right]=-8 \pi G\left(S_{i j}-\frac{1}{2} g_{i j} S\right),
$$

where $S_{i j}$ is the surface stress-energy tensor of $\Sigma$.

By regarding a thin shell as the limit of a layer of uniform finite thickness $\epsilon$ as $\epsilon \rightarrow 0$, we can give a heuristic justification for the name surface stressenergy tensor.

Let $\Sigma^{-}, \Sigma^{+}$be the two boundary surfaces separating the finite layer from the regions $M^{-}, M^{+}$. In gaussian coordinates the equations of $\Sigma^{-}$and $\Sigma^{+}$ are $x^{1}=0, x^{1}=\epsilon$.

In terms of the extrinsic curvature tensor and in the gaussian system of coordinates the Ricci tensor can be written as

$$
{ }^{4} R_{i j}=\frac{\partial K_{i j}}{\partial x^{1}}+Z_{i j},
$$

where

$$
Z_{i j}={ }^{3} R_{i j}-K K_{i j}+2 K_{i}^{p} K_{p j} .
$$

Integration of the Einstein field equations

$$
R_{\alpha \beta}=-8 \pi G\left(T_{\alpha \beta}-\frac{1}{2} g_{\alpha \beta} T\right),
$$

through the layer gives

$$
-8 \pi G \int_{0}^{\epsilon}\left(T_{i j}-\frac{1}{2} g_{i j} T\right) d x^{1}=\left[K_{i j}\right]+\int_{0}^{\epsilon} Z_{i j} d x^{1} .
$$


In the limit $\epsilon \rightarrow 0$ for fixed $K_{i j}^{-}, K_{i j}^{+}, K_{i j}$ remains bounded inside the layer. Hence, the integral of $Z_{i j}$ tends to zero. Comparing this result with (10) we see that

$$
S_{i j}=\lim _{\epsilon \rightarrow 0} \int_{0}^{\epsilon} T_{i j} d x^{1} .
$$

Thus, $S_{i j}$ is the integral of Einstein's energy tensor through the thickness of the layer.

\subsection{Matching Conditions from the Action}

We will consider now the problem of junction conditions in the context of $D$-dimensional theories [31]. In order to do this we will introduce the concept of domain wall. In a $D$-dimensional spacetime a domain wall can be defined as an extended object with $D-2$ spatial dimensions, which divides the spacetime in different domains. Here we will use this term to make reference to any $D-2$ brane moving in $D$ dimensions.

Let $M$ be a $D$-dimensional manifold containing a domain wall $\Sigma$, which splits $M$ into two parts $M^{-}$and $M^{+}$. The metric must be continuous everywhere, while its derivatives must be continuous everywhere except on $\Sigma$. We will denote $\Sigma^{ \pm}$as being the two sides of $\Sigma$.

Varying the Einstein-Hilbert action in $M^{ \pm}$gives ${ }^{1}$

$$
\delta S_{E H}=-\frac{1}{2} \int_{\Sigma^{ \pm}} d^{D-1} x \sqrt{-h} g^{M N} n^{P}\left(\nabla_{M} \delta g_{N P}-\nabla_{P} \delta g_{M N}\right),
$$

where $n_{M}$ is the unit normal pointing into $M^{ \pm}$and the induced metric on $\Sigma^{ \pm}$is given by the tangential components of the projection tensor $h_{M N}=$ $g_{M N}-n_{M} n_{N}$. If we contract the quantity in parenthesis with $n^{M} n^{N} n^{P}$, it vanishes, so we can replace $g^{M N}$ by $h^{M N}$.

The expression (16) contains a normal derivative of the metric variation, which is discontinuous across $\Sigma$ according to our initial hypothesis, therefore, the contributions from $M^{ \pm}$will not necessarily cancel out. In this way, it is necessary to add a Gibbons-Hawking boundary term [32] on each side of the domain wall to cancel out this term,

$$
S_{G H}=-\int_{\Sigma^{ \pm}} d^{D-1} x \sqrt{-h} K
$$

\footnotetext{
${ }^{1}$ For convenience we use units such that $8 \pi G=1$
} 
with $K$ being the trace of the extrinsic curvature of $\Sigma$, i.e. $K=h^{M N} K_{M N}$ where $K_{M N}=h_{M}^{P} h_{N}^{Q} \nabla_{P} n_{Q}$.

The variation of this new term is

$$
\delta S_{G H}=-\int_{\Sigma^{ \pm}} d^{D-1} x \sqrt{-h}\left(\delta K+\frac{1}{2} K h^{M N} \delta g_{M N}\right),
$$

where

$$
\delta K=-K^{M N} \delta g_{M N}-h^{M N} n^{P}\left(\nabla_{M} \delta g_{N P}-\frac{1}{2} \nabla_{P} \delta g_{M N}\right)+\frac{1}{2} K n^{P} n^{Q} \delta g_{P Q} .
$$

Thus, the total variation is

$$
\begin{aligned}
\delta S_{E H}+\delta S_{G H}= & \int_{\Sigma^{ \pm}} d^{D-1} x \sqrt{-h}\left[\frac{1}{2} h^{M N} n^{P} \nabla_{M} \delta g_{N P}+K^{M N} \delta g_{M N}-\right. \\
& \left.-\frac{1}{2} K n^{M} n^{N} \delta g_{M N}-\frac{1}{2} K h^{M N} \delta g_{M N}\right] .
\end{aligned}
$$

Notice that the first term in the rhs of (20) can be written as

$$
h^{M N} n^{P} \nabla_{M} \delta g_{N P}=\bar{\nabla}_{M}\left(h^{M N} n^{P} \delta g_{N P}\right)+K n^{M} n^{N} \delta g_{M N}-K^{M N} \delta g_{M N},
$$

where $\bar{\nabla}$ is the covariant derivative associated to $h$. We substitute this result into (20) to get

$$
\delta S_{E H}+\delta S_{G H}=\frac{1}{2} \int_{\Sigma^{ \pm}} d^{D-1} x \sqrt{-h}\left(K^{M N}-K h^{M N}\right) \delta g_{M N} .
$$

The domain wall has an action given by

$$
S_{d w}=\int_{\Sigma} d^{D-1} x \sqrt{-h} L_{d w}
$$

whose variation is

$$
\delta S_{d w}=\int_{\Sigma} d^{D-1} x \sqrt{-h} t^{M N} \delta g_{M N}
$$

where $t^{M N} \equiv \frac{2}{\sqrt{-h}} \frac{\delta S_{d w}}{\delta h_{M N}}$ is tangential to the domain wall such that we can replace $\delta h_{M N}$ by $\delta g_{M N}$.

The variation of the total action $S=S_{E H}+S_{G H}+S_{d w}$ gives the DarmoisIsrael conditions

$$
\left[K_{M N}-K h_{M N}\right]=-t_{M N},
$$

where the brackets stand for the jump of $K$ through the domain wall $\Sigma$. 


\subsection{Junction Conditions from Distribution Theory}

The Darmois-Israel conditions describe the motion of the domain wall through the bulk. However, sometimes we describe a static brane. In this case the Darmois-Israel conditions reduce to some relations between the energy density and pressure on the brane and the coefficients of the bulk metric. An alternative derivation of these relations can be done using distribution theory as follows.

Let us consider as an example a 5-dimensional bulk metric of the form $[33,34]$

$$
d s_{(5)}^{2}=-n^{2}(t, y) d t^{2}+a^{2}(t, y) \gamma_{i j} d x^{k} d x^{j}+b^{2}(t, y) d y^{2}
$$

where $\gamma_{i j}$ represents a maximally symmetric metric on the 3-brane located in $y=0$ with $k=-1,0,1$ parametrizing the spatial curvature.

The stress-energy tensor appearing in the Einstein's equations $G_{A B}=$ $\kappa_{(5)}^{2} \mathcal{T}_{A B}$ can be written as

$$
\mathcal{T}_{A B}=\hat{T}_{A B}+T_{A B}
$$

where $\hat{T}_{A B}$ is the stress-energy tensor of the matter on the bulk (and possibly other branes) which we do not need to specify here, and $T_{A B}$ corresponds to the matter content on the brane which can be expressed quite generally as

$$
T_{B}^{A}=\frac{\delta(y)}{b} \operatorname{diag}(-\rho, p, p, p, 0) .
$$

The energy $\rho$ and the pressure $p$ are independent of the position on the brane in order to recover standard cosmology on the brane.

From the metric (26) the Einstein's tensor components are found to be

$$
\begin{aligned}
\tilde{G}_{00}= & 3\left\{\frac{\dot{a}}{a}\left(\frac{\dot{a}}{a}+\frac{\dot{b}}{b}\right)-\frac{n^{2}}{b^{2}}\left(\frac{a^{\prime \prime}}{a}+\frac{a^{\prime}}{a}\left(\frac{a^{\prime}}{a}-\frac{b^{\prime}}{b}\right)\right)+k \frac{n^{2}}{a^{2}}\right\} \\
\tilde{G}_{i j}= & \frac{a^{2}}{b^{2}} \gamma_{i j}\left\{\frac{a^{\prime}}{a}\left(\frac{a^{\prime}}{a}+2 \frac{n^{\prime}}{n}\right)-\frac{b^{\prime}}{b}\left(\frac{n^{\prime}}{n}+2 \frac{a^{\prime}}{a}\right)+2 \frac{a^{\prime \prime}}{a}+\frac{n^{\prime \prime}}{n}\right\}+ \\
& +\frac{a^{2}}{n^{2}} \gamma_{i j}\left\{\frac{\dot{a}}{a}\left(-\frac{\dot{a}}{a}+2 \frac{\dot{n}}{n}\right)-2 \frac{\ddot{a}}{a}+\frac{\dot{b}}{b}\left(-2 \frac{\dot{a}}{a}+\frac{\dot{n}}{n}\right)-\frac{\ddot{b}}{b}\right\}-k \gamma_{i j}, \\
\tilde{G}_{05}= & 3\left(\frac{n^{\prime}}{n} \frac{\dot{a}}{a}+\frac{a^{\prime}}{a} \frac{\dot{b}}{b}-\frac{\dot{a}^{\prime}}{a}\right)
\end{aligned}
$$




$$
\tilde{G}_{55}=3\left\{\frac{a^{\prime}}{a}\left(\frac{a^{\prime}}{a}+\frac{n^{\prime}}{n}\right)-\frac{b^{2}}{n^{2}}\left(\frac{\dot{a}}{a}\left(\frac{\dot{a}}{a}-\frac{\dot{n}}{n}\right)+\frac{\ddot{a}}{a}\right)-k \frac{b^{2}}{a^{2}}\right\},
$$

where a prime stands for derivative with respect to $y$ and the dot means derivative with respect to $t$.

In order to have a well defined geometry the metric must be continuous across the brane. However, its derivatives with respect to $y$ can be discontinuous in $y=0$. Thus, there is a Dirac delta function in the second derivatives of the metric with respect to $y$. In general we will have

$$
a^{\prime \prime}=\hat{a}^{\prime \prime}+\left[a^{\prime}\right] \delta(y)
$$

where $\hat{a}^{\prime \prime}$ stands for the non-distributional part of the second derivative of $a$ (the ordinary second derivative), and $\left[a^{\prime}\right]$ is the jump of the first derivative across $y=0$ defined by

$$
\left[a^{\prime}\right]=a^{\prime}\left(0^{+}\right)-a^{\prime}\left(0^{-}\right) .
$$

The resulting terms with a delta function appearing in the Einstein tensor must match the distributional part of the stress-energy tensor in order to satisfy the Einstein's equations. Comparing the Dirac delta functions in the components (29) and (30) of the Einstein's tensor we obtain the relations

$$
\begin{aligned}
\frac{\left[a^{\prime}\right]}{a_{0} b_{0}} & =-\frac{\kappa_{(5)}^{2}}{3} \rho, \\
\frac{\left[n^{\prime}\right]}{n_{0} b_{0}} & =\frac{\kappa_{(5)}^{2}}{3}(3 p+2 \rho),
\end{aligned}
$$

where the subscript 0 means that the metric coefficients take their values on the brane.

\section{The Scenarios}

We shall consider two kinds of scenarios. First, a brane wall model based on the exact solution of Einstein Equations and boundary conditions, where the brane is a so-called domain wall embedded in a space-time containing a singularity (a type of black hole) and a cosmological constant. Subsequently we consider a membrane of the Friedmann-Robertson-Walker type. 


\subsection{Brane Wall Model}

In the first case we have a scenario described by the gravitational action in a D-dimensional bulk with a scalar field (the bulk dilaton), a domain wall potential and a Gibbons-Hawking term [32],

$$
S=\int_{b u l k} d^{D} x \sqrt{-g}\left(\frac{1}{2} R-\frac{1}{2}(\partial \phi)^{2}-V(\phi)\right)-\int_{d w} d^{D-1} x \sqrt{-h}([K]+\hat{V}(\phi)),
$$

where $\phi$ is the bulk dilaton, $K$ is the extrinsic curvature, $V(\phi)$ and $\hat{V}(\phi)$ are bulk and domain wall potentials respectively, and $g$ and $h$ denote the bulk and domain wall metrics. The potentials are here:

$$
\begin{aligned}
& V(\phi)=V_{0} e^{\beta \phi} \\
& \hat{V}(\phi)=\hat{V}_{0} e^{\alpha \phi} .
\end{aligned}
$$

We consider the bulk metric as being static and invariant under rotation,

$$
d s^{2}=-U(r) d t^{2}+U(r)^{-1} d r^{2}+R(r)^{2} d \Omega_{k}^{2},
$$

where $d \Omega_{k}^{2}$ is the line element on a $D-2$ dimensional space of constant curvature depending on a parameter $k$. We can also consider the brane to have a static metric, in which case the solution of the bulk would be more complicated. The above metric is supposed to have a mirror symmetry $Z_{2}$ with respect to the domain wall. Such a symmetry will be used in order to impose the Darmois-Israel conditions. The variation of the total action (36) including the Gibbons-Hawking term leads directly to

$$
K_{M N}=-\frac{1}{2(D-2)} \hat{V}(\phi) h_{M N}
$$

The extrinsic curvature can be computed as

$$
K_{M N}=h_{M}^{P} h_{N}^{Q} \nabla_{P} n_{Q}
$$

where the unit normal, which points into $r<r(t)$, is

$$
n_{M}=\frac{1}{\sqrt{U-\frac{\dot{r}^{2}}{U}}}(\dot{r},-1,0 \ldots, 0) .
$$


Here a dot means derivative with respect to the bulk time $t$.

The $i j$ component of (40) can be written as

$$
\frac{R^{\prime}}{R}=\frac{\hat{V}(\phi)}{2(D-2) U} \sqrt{U-\frac{\dot{r}^{2}}{U}},
$$

while the 00 component is

$$
\left(\frac{R^{\prime}}{R}\right)^{-1}\left(\frac{R^{\prime}}{R}\right)^{\prime}=\frac{\hat{V}^{\prime}(\phi)}{\hat{V}(\phi)}-\frac{R^{\prime}}{R} .
$$

Here a prime denotes derivative with respect to the extra coordinate $r$.

The equation of motion for the dilaton obtained from the action (36), together with (44), can be simultaneously solved with the Ansatz (39), leading to $[31]$

$$
\begin{aligned}
\phi(r) & =\phi_{\star}-\frac{\alpha(D-2)}{\alpha^{2}(D-2)+1} \log r \\
R(r) & =\left(\alpha^{2}(D-2)+1\right) C \hat{V}_{0} e^{\alpha \phi_{\star}} r^{\frac{1}{\alpha^{2}(D-2)+1}}
\end{aligned}
$$

where $\phi_{\star}$ and $C$ are arbitrary integration constants.

The motion of the domain wall is governed by the $i j$ component of the Darmois-Israel conditions (43). That equation can be written in terms of the brane proper time $\tau$ as

$$
\frac{1}{2}\left(\frac{d R}{d \tau}\right)^{2}+F(R)=0
$$

The induced metric on the domain wall is Friedmann-Robertson-Walker and (47) describes the evolution of the scale factor $R(\tau)$. This equation is the same as that one for a particle of unit mass and zero energy rolling in a potential $F(R)$ given by

$$
F(R)=\frac{1}{2} U R^{\prime 2}-\frac{1}{8(D-2)^{2}} \hat{V}^{2} R^{2} .
$$

Notice that the solution only exists when $F(R) \leq 0$. 
From the induced domain wall metric we find the relations between the time parameter on the brane $(\tau)$ and in the bulk $(t)$ as given by

$$
d t=\frac{\sqrt{U+\left(\frac{d r}{d \tau}\right)^{2}}}{U} d \tau
$$

so that

$$
\dot{r} \equiv \frac{d r}{d t}=\frac{d r}{d \tau} \frac{d \tau}{d t}=\frac{d r}{d \tau} \frac{U}{\sqrt{U+\left(\frac{d r}{d \tau}\right)^{2}}},
$$

where $\frac{d r}{d \tau}=\frac{d R}{d \tau}\left(\frac{d R}{d r}\right)^{-1}$ can be obtained from (47). Equation (49) describes the motion of a domain wall in the static background as seen by an observer in the bulk.

Consider two points on the brane. In general, there are more than one null geodesic connecting them in the D-dimensional spacetime. The trajectories of photons must be on the brane and those of gravitons may be outside. We consider the shortest path for both photons and gravitons. For the latter, the geodesic equation is the same as the one considered in [11], since the bulk metric is static:

$$
\ddot{r}_{g}+\left(\frac{1}{r_{g}}-\frac{3}{2} \frac{U^{\prime}}{U}\right) \dot{r}_{g}^{2}+\frac{1}{2} U U^{\prime}-\frac{U^{2}}{r_{g}}=0 .
$$

Again a dot means derivative with respect to the bulk time $t$.

The solutions of (49) and (50) in terms of the bulk proper time $t$ were obtained by means of a MAPLE program. In the next section we discuss the possibility of shortcuts in the cases of the various solutions describing different Universes defined by the domain wall solution.

\subsection{Cosmological Brane Model}

Later, we consider a scenario where the bulk is a purely Anti-de-Sitter spacetime of the form [35],

$$
d s^{2}=h(a) d t^{2}-\frac{d a^{2}}{h(a)}-a^{2} d \Sigma^{2},
$$


with $h(a)=k+\frac{a^{2}}{l^{2}}, l \sim 0.1 \mathrm{~mm}$ is the Randall-Sundrum lenght scale [36] and $d \Sigma^{2}$ represents the metric of the three dimensional spatial sections with $k=0, \pm 1$,

$$
d \Sigma^{2}=\frac{d r^{2}}{1-k r^{2}}+r^{2}\left[d \theta^{2}+\sin ^{2}(\theta) d \phi^{2}\right] .
$$

The brane is localized at $a_{b}(\tau)$, where $\tau$ is the proper time on the brane. The unit vector normal to the brane is defined as (overdot and prime superscript denote differentiation with respect to $\tau$ and $a$ respectively)

$$
n=\dot{a}_{b}(\tau) d t-\dot{t}(\tau) d a \text {. }
$$

The normalization of $n$ implies the relation between the bulk time $t$ and the brane time $\tau$,

$$
h\left(a_{b}\right) \dot{t}^{2}-\dot{a}_{b}^{2} h^{-1}\left(a_{b}\right)=1
$$

and also the usual FRW expression for the distance on the brane ,

$$
d s^{2}=h_{i j} d x^{i} d x^{j}=d \tau^{2}-a_{b}^{2} d \Sigma^{2} .
$$

We also need the second fundamental form $K_{i j}=e_{i}^{\alpha} e_{j}^{\nu} \nabla_{\alpha} n_{\nu}$, given by

$$
\begin{aligned}
K_{r r} & =-\frac{a_{b} h}{\left(1-k r^{2}\right)} \dot{t}, \\
K_{\theta \theta} & =-a_{b} r^{2} h \dot{t}, \\
K_{\phi \phi} & =-a_{b} r^{2} \sin ^{2}(\theta) h \dot{t} \\
K_{\tau \tau} & =\frac{1}{h \dot{t}}\left(\ddot{a}_{b}+\frac{h^{\prime}}{2}\right) .
\end{aligned}
$$

We now use the Darmois-Israel conditions for a $Z_{2}$ symmetric configuration (25),

$$
K_{i j}=\frac{1}{2} \kappa_{(5)}^{2}\left(S_{i j}-\frac{1}{3} h_{i j} S\right)
$$

with an isotropic distribution of matter,

$$
S_{i j}=\epsilon_{T} u_{i} u_{j}-p_{T}\left(h_{i j}-u_{i} u_{j}\right) \quad .
$$

Thus, the following relations hold [37],[38], [33]

$$
\begin{aligned}
\frac{d \epsilon_{T}}{d \tau} & =-3 \frac{\dot{a}_{b}}{a_{b}}\left(\epsilon_{T}+p_{T}\right), \\
\frac{\dot{a}_{b}^{2}}{a_{b}^{2}}+\frac{h}{a_{b}^{2}} & =\frac{\kappa_{(5)}^{4} \epsilon_{T}^{2}}{36} .
\end{aligned}
$$


Following [39] and [40], we introduce an intrinsic non-dynamical energy density $\epsilon_{0}$ defined by means of $\epsilon_{T}=\epsilon_{0}+\epsilon, p_{T}=-\epsilon_{0}+p$ where $\epsilon$ and $p$ correspond to the brane matter. Thus, the junction equations imply the usual energy conservation on the brane,

$$
\frac{d \epsilon}{d \tau}=-3 \frac{\dot{a}_{b}}{a_{b}}(\epsilon+p)
$$

and the modified Friedmann equation [34],

$$
H^{2}=\left(\frac{\dot{a}_{b}}{a_{b}}\right)^{2}=\frac{\Lambda_{4}}{3}+\frac{1}{M_{P l}^{2}}\left(\frac{\epsilon}{3}+\frac{\epsilon^{2}}{6 \epsilon_{0}}\right)-\frac{k}{a_{b}^{2}},
$$

with the hierarchy

$$
M_{P L}^{-2}=\frac{\kappa_{5}^{4} \epsilon_{0}}{6}
$$

and the cosmological constant on the brane,

$$
\frac{\Lambda_{4}}{3}=\left(\frac{\kappa_{5}^{4} \epsilon_{0}^{2}}{36}-\frac{1}{l^{2}}\right)
$$

The present density of the Universe is

$$
\epsilon(0)=\Omega_{0} \epsilon_{c}=\Omega_{0} 3 M_{P L}^{2} H_{0}^{2},
$$

where $\Omega_{0}$ is the ratio between the density and the critical density of the Universe. Aiming at the energy conservation, the Friedmann equation is

$$
H^{2}=\frac{\Lambda_{4}}{3}+\Omega_{0} H_{0}^{2} \frac{a_{b 0}^{q}}{a_{b}^{q}}\left(1+\frac{\Omega_{0}}{4\left(1+\Lambda_{4} l^{2} / 3\right)} \frac{L_{c}^{q}}{a_{b}^{q}}\right)-\frac{k}{a_{b}^{2}},
$$

with $L_{c}^{q}=a_{b 0}^{q} l^{2} H_{0}^{2}$.

We thus verify that there exist three phases in the evolution of the Universe. When $a_{b}>>L_{c}$, the linear term in the energy density prevails in the Friedmann equation, leading to the standard cosmology. Because $H_{0} l \sim 10^{-29}$ this happens in a redshift of $a_{b 0} / a_{b} \sim 10^{15}$, much earlier than the nucleosynthesis. For $a_{b}<<L_{c}$ the Universe expands at a slower pace as compared to the standard model, $a_{b} \propto \tau^{1 / q}$, and the quadratic term is the prevailing one. In an intermediate era where $a_{b} \sim L_{c}$, both phases coexist. 
We see that all the cosmological information is already known: the position of the brane in the extra dimension, $a_{b}(\tau)$, is just the scale factor of the FRW metric and the junction conditions imply that the cosmological evolution of the brane is obtained by the usual energy conservation (61) and the modified Friedmann equations (62). However, in this work we are particularly interested in the evolution of the brane with respect to the bulk. This relation can be obtained using (53) and transforming from the time of the brane to the time of the bulk. Thus, the position of the brane can also be treated as a function of the bulk proper time satisfying the equation

$$
\frac{d a_{b}}{d t}=\frac{d a_{b}}{d \tau} \frac{d \tau}{d t}=\dot{a}_{b}(\tau) \frac{h\left(a_{b}\right)}{\sqrt{h\left(a_{b}\right)+\dot{a}_{b}(\tau)^{2}}} .
$$

\section{Solutions of the Geodesic Equation for a Domain Wall}

\section{$5.1 \quad$ Type I Solutions}

We define the type I brane solutions as those for which $\alpha=\beta=0$. Consequently, the potentials become cosmological constants. The solution also has a constant dilaton $\phi=\phi_{0}$. A simple rescaling in the metric leads us to

$$
d s^{2}=-U(R) d t^{2}+U(R)^{-1} d R^{2}+R^{2} d \Omega_{k}^{2},
$$

with

$$
U(R)=k-2 M R^{-(D-3)}-\frac{2 V_{0}}{(D-1)(D-2)} R^{2},
$$

which corresponds to a topological black hole solution in D dimensions with a cosmological constant.

As discussed in [31], if the domain wall has positive energy density $\left(\hat{V}_{0}>\right.$ 0 ), the relevant part of the bulk spacetime is $R<R(\tau)$, which is the region containing the singularity. If it has negative energy density, the relevant part is $R>R(\tau)$, which is non-singular unless the wall reaches $R=0$.

The potential $F(R)$ ruling the evolution of the scale factor is

$$
F(R)=\frac{k}{2}-M R^{-(D-3)}-\hat{\Lambda} R^{2}
$$




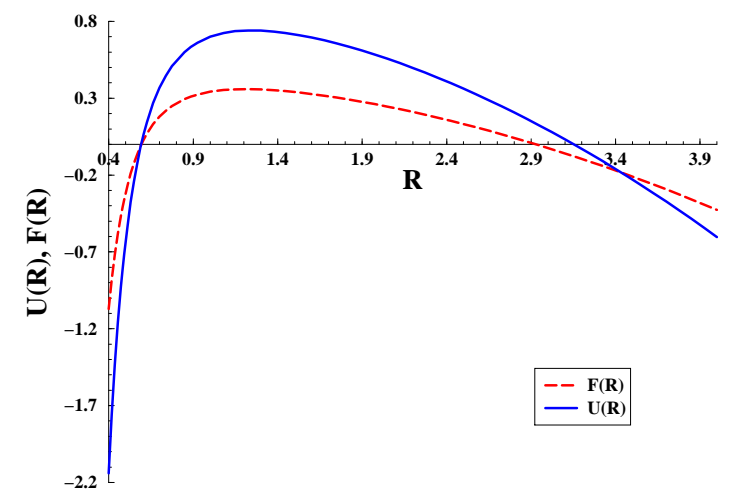

(a)

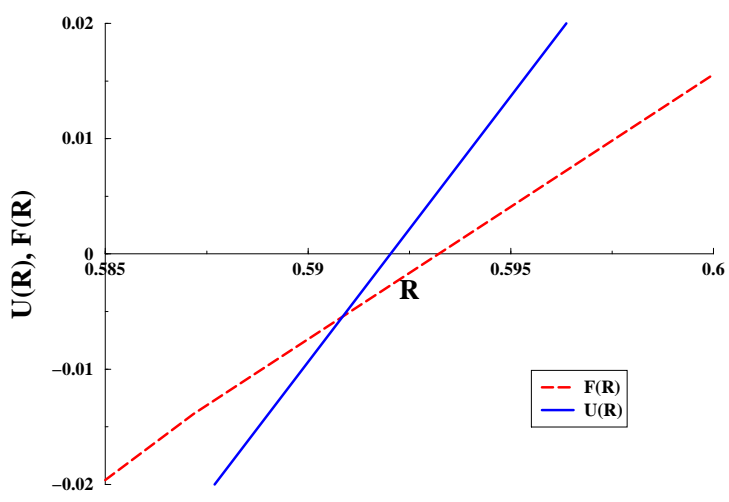

(b)

Figure 1: (a) $U(R)$ and $F(R)$ for type I solutions with $M=1 / 10, V_{0}=1$ and $\hat{V}_{0}= \pm 1$, (b) Zoom of the event horizon region.

where the effective cosmological constant on the domain wall is given by

$$
\hat{\Lambda}=\frac{1}{D-2}\left[\frac{V_{0}}{D-1}+\frac{\hat{V}_{0}^{2}}{8(D-2)}\right] .
$$

We shall analyze each of the four cases presented in [31]. As we have previously stated, the equation of motion (47) has a solution only when $F(R) \leq 0$. This is automatic if $U(R)<0$, i.e. if $r$ is a time coordinate; therefore, we look for solutions with $U(R)>0$. In fact, both conditions,

$$
F(R) \leq 0 \quad \text { and } \quad U(R)>0
$$

can coexist in some cases as we will see in what follows. In order to ilustrate the following examples we have chosen $D=6$ dimensions.

\subsection{1 $\hat{\Lambda}>0, M>0$}

From the graph of $U(R)$ (see Fig.1) we can choose the initial condition for the domain wall assuming that (68) describes a dS-Schwarzschild bulk with event and cosmological horizons when $M>0$ and $V_{0}>0$. 


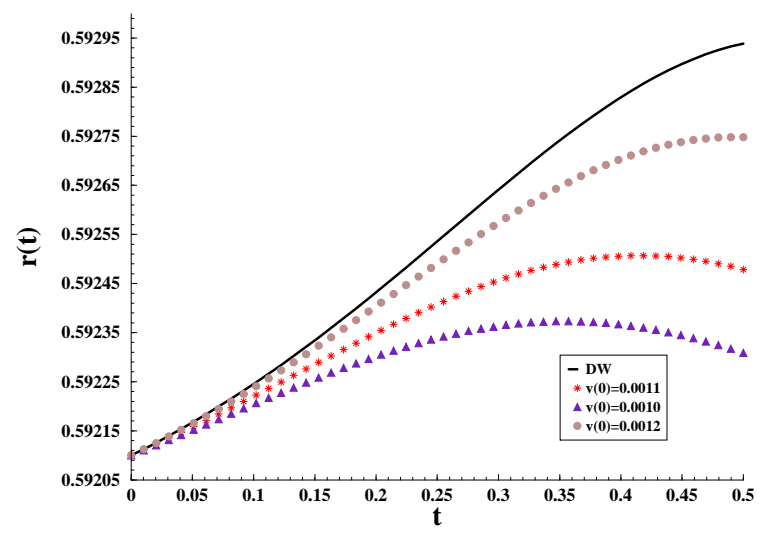

(a)

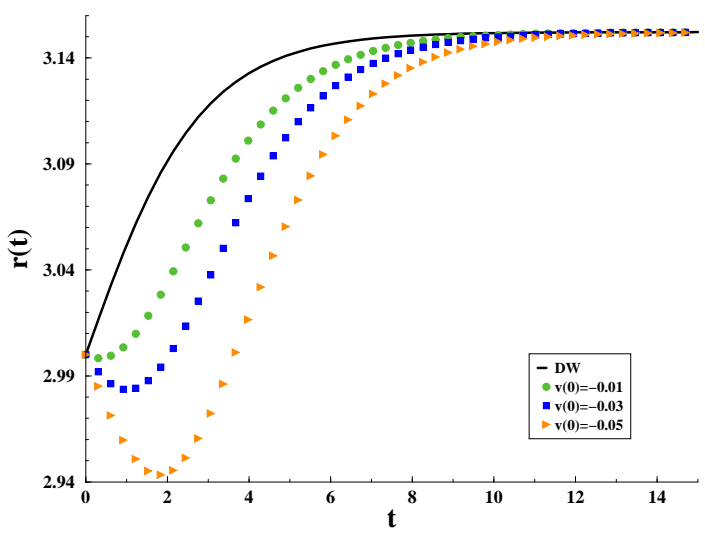

(b)

Figure 2: Domain wall motion and geodesics for type I solutions with $M=$ $1 / 10, V_{0}=1$ and $\hat{V}_{0}=1$ in (a) region I and, (b) region II.

We thus choose the initial condition for the domain wall inside this region and where $r$ is a space coordinate. From Fig.1 we notice that there are two small regions, $r_{H} \leq r<0.593$ and $2.93 \leq r<r_{C}$, where (71) holds. The results are shown in Fig.2. We see that for region I the geodesics follow the domain wall for a while and then decouple falling into the event horizon. For region II all the geodesics and the domain wall converge to the cosmological horizon $r_{C}$ independently of the value of $\hat{V}_{0}$.

\subsection{2 $\hat{\Lambda}<0, M>0$}

This is an AdS-Schwarzschild bulk. The condition (71) is fullfilled inside a very small range as we can see in Fig.3(a). However, all the geodesics fall into the event horizon after following some path on the brane (see Fig.3(b)).

\subsection{3 $\hat{\Lambda}>0, M<0$}

From Fig.4(a) we choose the initial condition for the domain wall equation of motion inside the region where (71) holds. As we can see from Fig.4(b), 


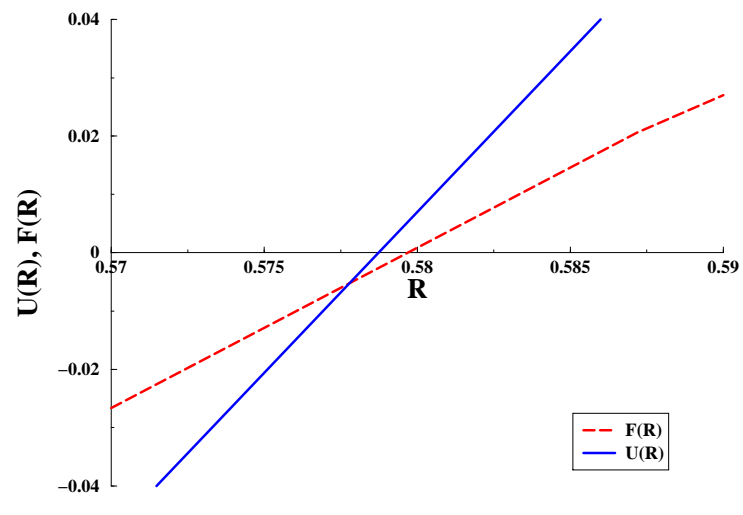

(a)

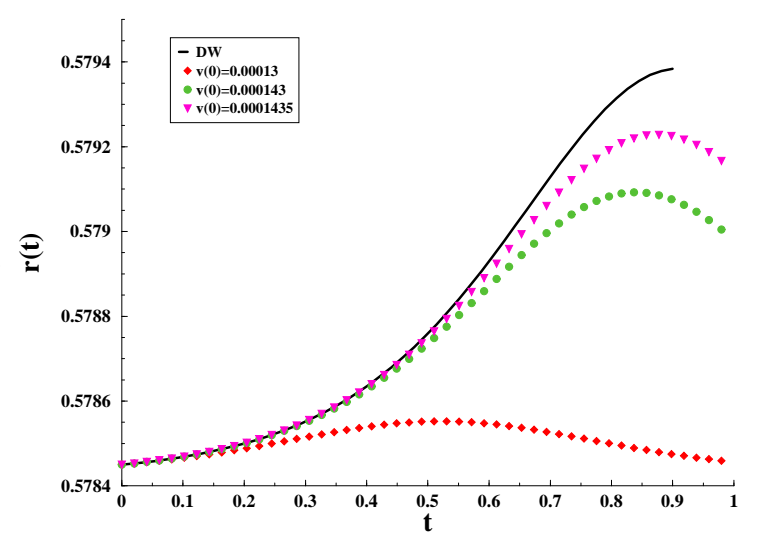

(b)

Figure 3: (a) Zoom of the region where (71) holds from the graph of $U(R)$ and $F(R)$ with $\hat{\Lambda}<0$ and $M>0$ for type I solutions, (b) Domain wall motion and geodesics for type I solutions with $M=1 / 10, V_{0}=-1$ and $\hat{V}_{0}=1$.

the domain wall and the geodesics converge to the cosmological horizon $r_{C}$. However, after some threshold initial velocity the geodesics diverge to the timelike naked singularity.

\subsection{4 $\hat{\Lambda}<0, M<0$}

Here (47) can only have a solution when $k=-1$. This is a topological black hole in an asymptotically AdS space. From Fig.5 we see that there is no solution fulfilling (71) between event and cosmological horizons.

\subsection{Type II Solutions}

The type II solutions have $\alpha=\beta / 2$ and $k=0$. The metric is given by

$$
U(r)=\left(1+b^{2}\right)^{2} r^{\frac{2}{1+b^{2}}}\left(-2 M r^{-\frac{D-1-b^{2}}{1+b^{2}}}-\frac{2 \Lambda}{\left(D-1-b^{2}\right)}\right),
$$




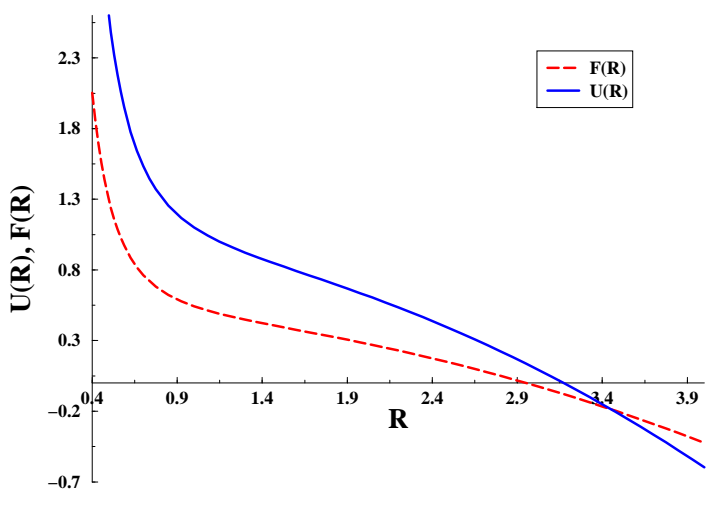

(a)

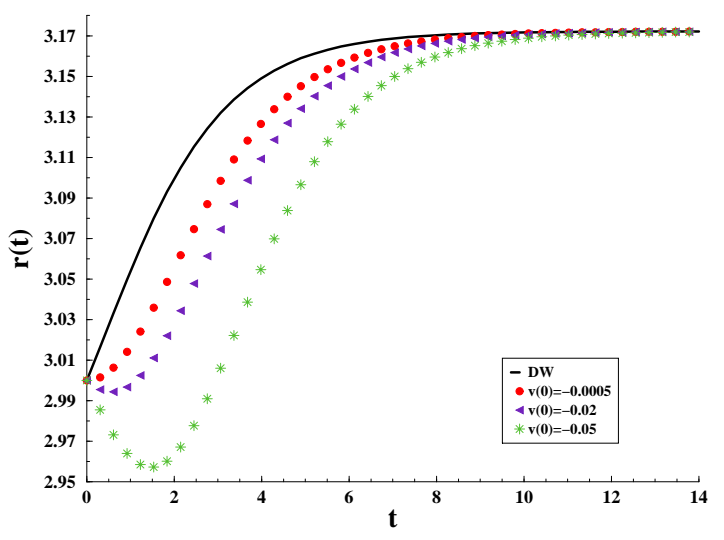

(b)

Figure 4: (a) $U(R)$ and $F(R)$ with $\hat{\Lambda}>0$ and $M<0$ for type I solutions, (b) Domain wall motion and geodesics for $M=-1 / 10, V_{0}=1$ and $\hat{V}_{0}=1$.

and the scale factor is

$$
R(r)=r^{\frac{1}{1+b^{2}}}
$$

where

$$
\Lambda=\frac{V_{0} e^{2 b \phi_{0}}}{D-2} \quad \text { and } \quad b=\frac{1}{2} \beta \sqrt{D-2} .
$$

The potential is given by the expression

$$
F(R)=-R^{2\left(1-b^{2}\right)}\left(M R^{-\left(D-1-b^{2}\right)}+\hat{\Lambda}\right),
$$

where

$$
\hat{\Lambda}=\frac{e^{2 b \phi_{0}}}{D-2}\left(\frac{V_{0}}{D-1-b^{2}}+\frac{\hat{V}_{0}^{2}}{8(D-2)}\right) .
$$

There are twelve cases from which we choose those ones where $r$ is a spatial coordinate. When $b^{2}<D-1, r$ is a spatial coordinate if $V_{0}<0$. When $b^{2}>D-1, r$ is spatial if $M<0$.

We should also rewrite $(71)$ as

$$
F(r) \leq 0 \text { and } \quad U(r)>0 .
$$




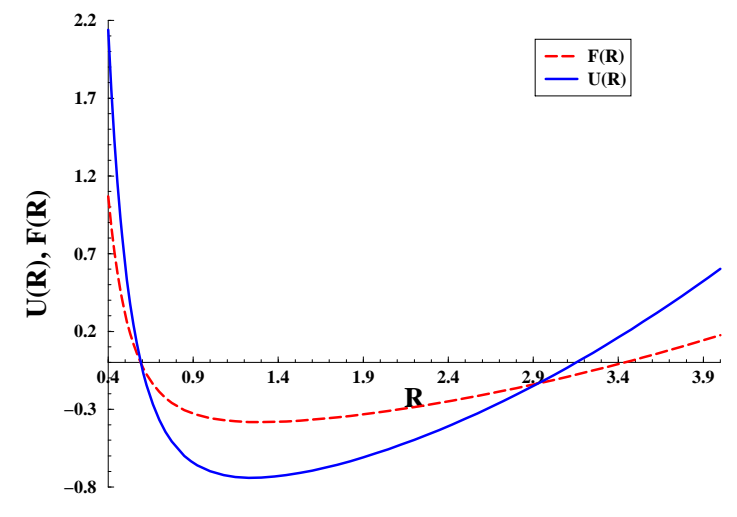

(a)

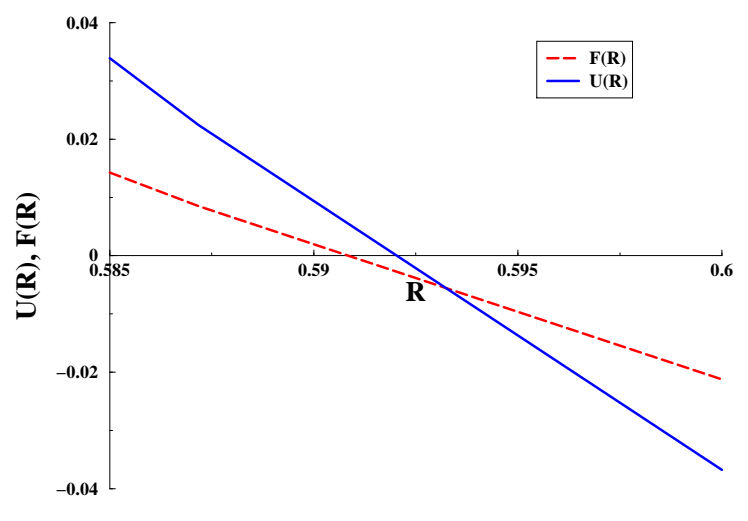

(b)

Figure 5: (a) $U(R)$ and $F(R)$ with $\hat{\Lambda}<0$ and $M=-1 / 10$ for type I solutions, (b) Zoom of the event horizon region.

\subsection{1 $\hat{\Lambda}>0, M<0, b^{2}>D-1$}

In this case $U(r)$ is always positive, whereas $F(r)$ is negative for small $r$. From Fig. 6 we see that some microscopic shortcuts appear in the very beginning of the solution and after crossing the domain wall they escape to infinity.

\subsection{2 $\hat{\Lambda}<0, M>0, b^{2}<1$}

This case describes a black $(D-2)$ brane solution in AdS space. Here there is a very small region where (77) holds after the event horizon as we can see from Fig.7. We show the entire domain wall solution and we see that geodesics follow it and then fall into the event horizon at later times.

\subsection{3 $\hat{\Lambda}<0, M>0,1<b^{2}<D-1$}

This case is also a black brane in AdS space. The region where (77) is respected is shown in Fig.8. As in the previous case all the geodesics follow the domain wall and at later times fall into the event horizon. 


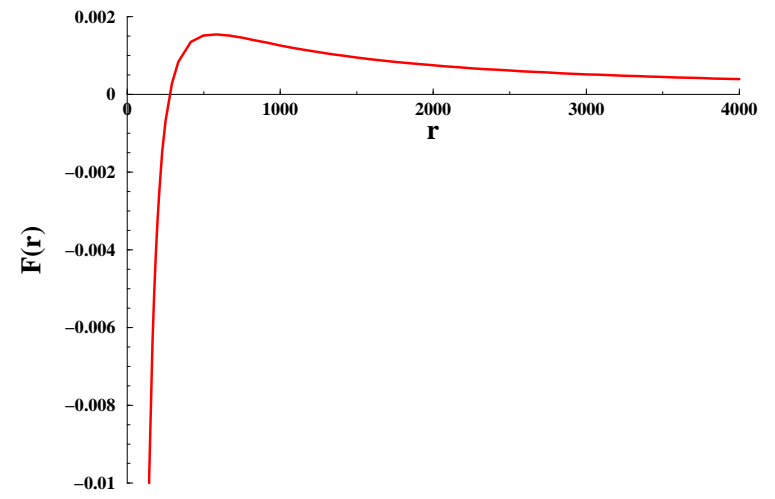

(a)

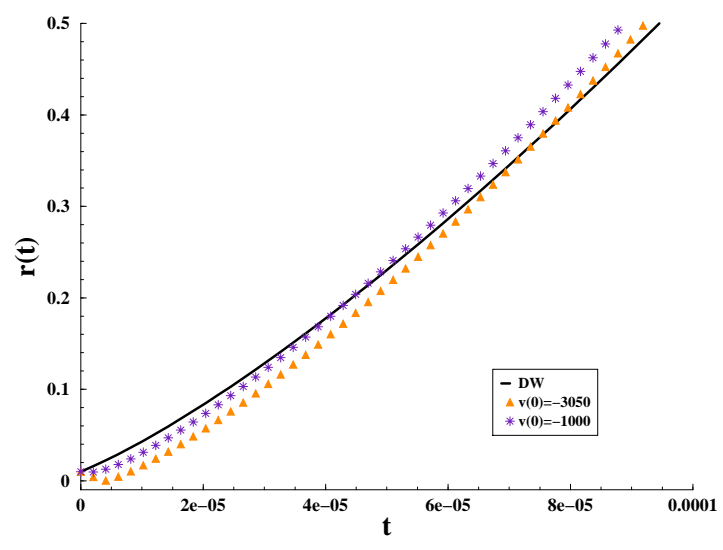

(b)

Figure 6: (a) $F(r)$ with $\hat{\Lambda}>0$ and $M<0$ for type II solutions, (b) Domain wall motion and geodesics for $V_{0}=1, \hat{V}_{0}=6, M=-10$ and $\beta=\sqrt{10}$.

5.2.4 $\hat{\Lambda}<0, M<0$

As $F(r)$ is always positive for all $b^{2}$, no solutions to (49) exist.

\subsection{Type III Solutions}

The type III solutions have $\alpha=\frac{2}{\beta(D-2)}$. In this case, the metric is given by

$$
U(r)=\left(1+b^{2}\right)^{2} r^{\frac{2}{1+b^{2}}}\left(-2 M r^{\frac{-1+b^{2}(D-3)}{1+b^{2}}}-\frac{2 \Lambda}{\left(1+b^{2}(D-3)\right)}\right),
$$

and the scale factor is

$$
R(r)=\gamma r^{\frac{b^{2}}{1+b^{2}}}
$$

where $\gamma=\left(\frac{(D-3)}{2 k \Lambda\left(1-b^{2}\right)}\right)^{1 / 2}$. The values of $\Lambda$ and $b$ are the same as those given in (74). 


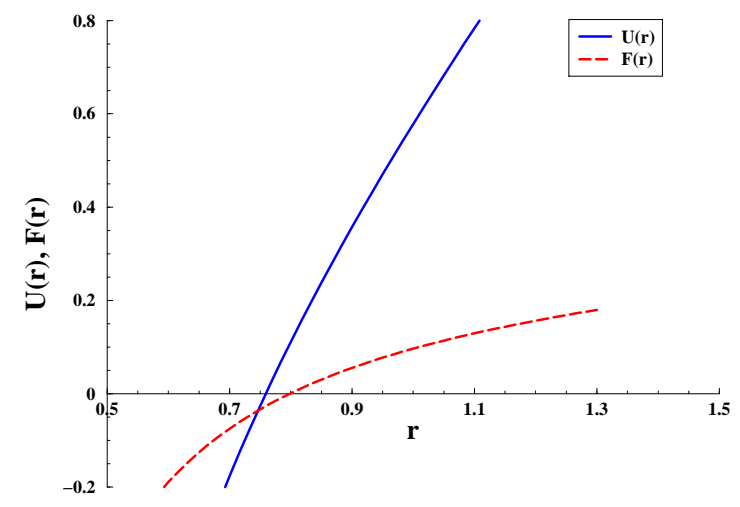

(a)

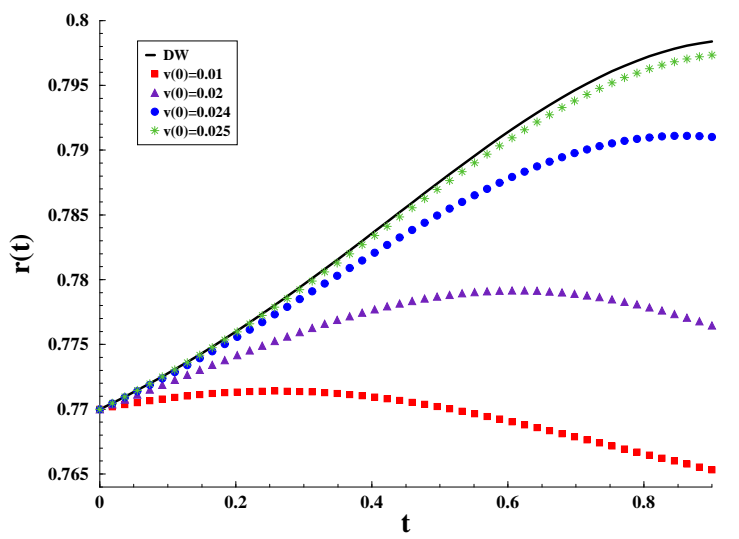

(b)

Figure 7: (a) $U(r)$ and $F(r)$ with $\hat{\Lambda}<0$ and $M>0$ for type II solutions, (b) Domain wall motion and geodesics for $V_{0}=-1, \hat{V}_{0}=1, M=1 / 10$ and $\beta=1 / \sqrt{2}$.

The potential $F(R)$ is

$$
\begin{aligned}
F(R)= & -\frac{(D-3) b^{4}}{2 k\left(1-b^{2}\right)\left(1+b^{2}(D-3)\right)}-M \gamma^{2} b^{4}\left(\frac{R}{\gamma}\right)^{-\left(D-3+\frac{1}{b^{2}}\right)}- \\
& -\frac{\hat{V}_{0}^{2} e^{\frac{2 \phi_{0}}{b}} \gamma^{2}}{8(D-2)^{2}}\left(\frac{R}{\gamma}\right)^{-2\left(\frac{1}{b^{2}}-1\right)} .
\end{aligned}
$$

If $V_{0}>0, r$ turns out to be a time coordinate, while for $V_{0}<0$, it is a spatial coordinate. From the twelve cases shown in [31] we choose those where it is a spatial coordinate. The condition (77) always applies.

\subsection{1 $V_{0}<0, M>0, b^{2}<\frac{1}{(D-1)}$}

This case describes a topological black hole in AdS space. From Fig.9 we can see the region where (77) holds. There are no shortcuts in this interval. 


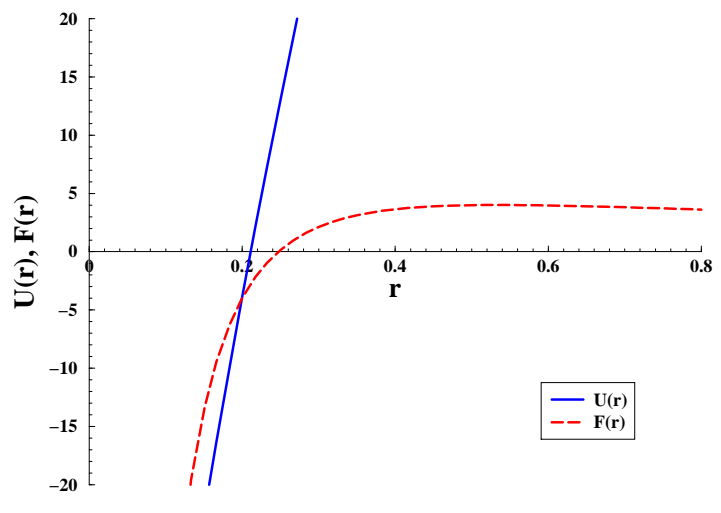

(a)

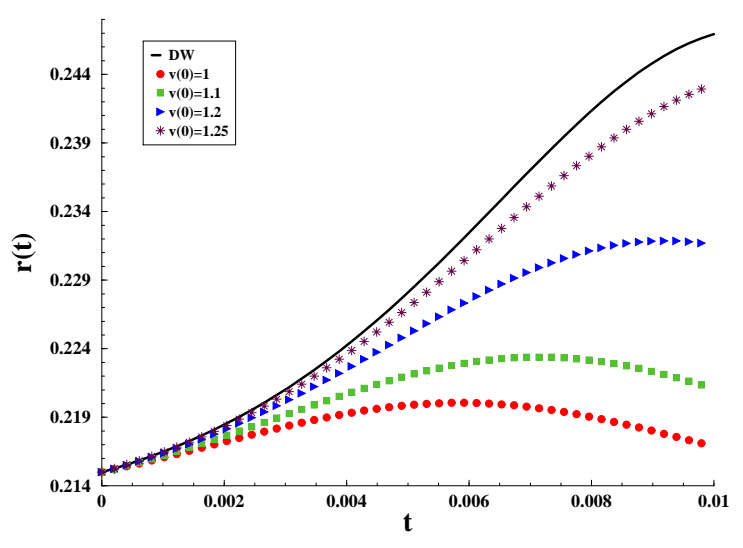

(b)

Figure 8: (a) $U(r)$ and $F(r)$ with $\hat{\Lambda}<0$ and $M>0$ for type II solutions, (b) Domain wall motion and geodesics for $V_{0}=-1, \hat{V}_{0}=1, M=10$ and $\beta=2$.

\subsection{2 $V_{0}<0, M>0, \frac{1}{(D-1)}<b^{2}<1$}

We again have a topological black hole in AdS space. There is a small interval where (49) has solution as we can see from Fig.10(a). Our results are shown in Fig.10(b). Notice that the domain wall equation of motion has a solution only inside the interval shown there. This means that only a group of geodesics with initial velocity $\dot{r}(0)>v_{c}$ can meet the domain wall after a roundabout in the bulk.

\subsection{3 $V_{0}<0, M>0, b^{2}>1$}

The black hole in AdS space appearing here has round spatial section. In this case $U(r)$ is always positive (then $r$ is always a spatial coordinate); however, we notice that $F(r) \leq 0$ for $r \geq 3 * 10^{5}$. We found that shortcuts are possible for several initial velocities if $M=0$.

The case $M>0$ is shown in Fig.11. We have two regions of interest after the event horizon depending only on the sign of $F(r)$ since $U(r)$ is positive in this range. In the first region all the geodesics initially follow the brane 


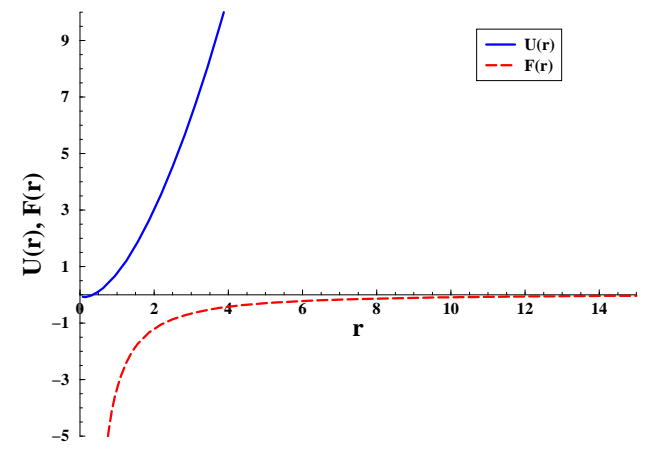

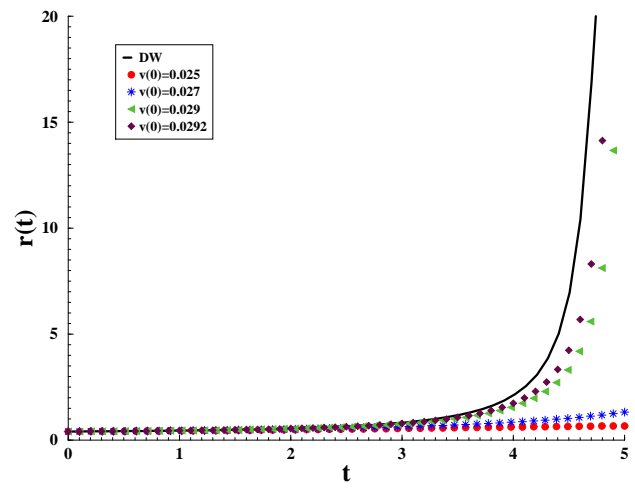

(b)

Figure 9: (a) $U(r)$ and $F(r)$ for Type III solutions with $k=-1, M=1 / 10$ and $\beta^{2}<\frac{1}{(D-2)}$, (b) Domain Wall motion and geodesics for $V_{0}=-1, \hat{V}_{0}=1$, $\phi_{0}=1$ and $\beta=1 / \sqrt{6}$.

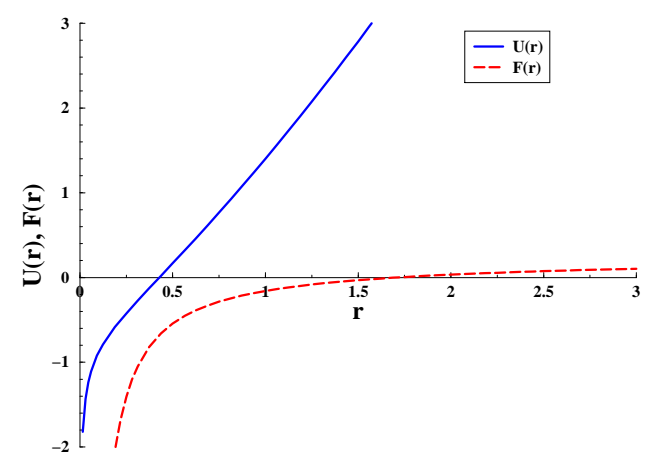

(a)

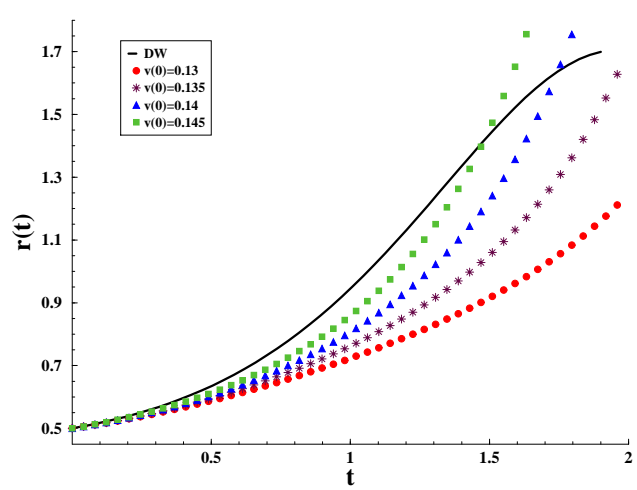

(b)

Figure 10: (a) $U(r)$ and $F(r)$ for $k=-1, M=1 / 10$ and $V_{0}<0$ in Type III solutions, (b) Domain wall motion and geodesics with $V_{0}=-1, \hat{V}_{0}=1$, $\phi_{0}=1$ and $\beta=1 / \sqrt{2}$. 

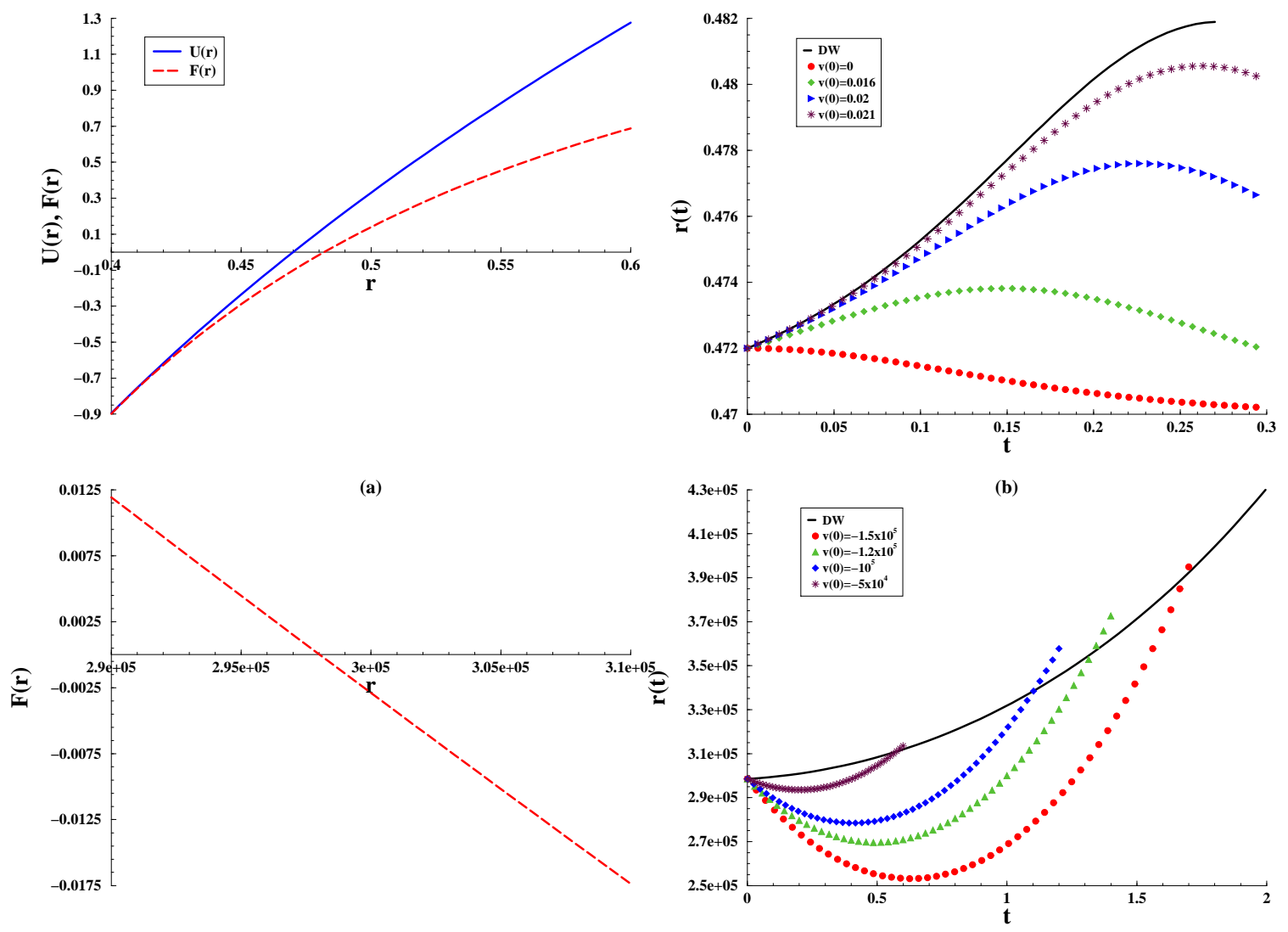

(c)

(d)

Figure 11: (a) $U(r)$ and $F(r)$ for $M=1 / 10$ and $V_{0}<0$ in Type III solutions, (b) Domain wall motion and geodesics with $V_{0}=-1, \hat{V}_{0}=1, \phi_{0}=1$ and $\beta=\sqrt{5} / 2$, (c) $F(r)$ in the region of interest, (d) Domain wall motion and geodesics under the same conditions as (b).

and fall into the event horizon at later times. In the second region we have shortcuts again for several initial velocities. 


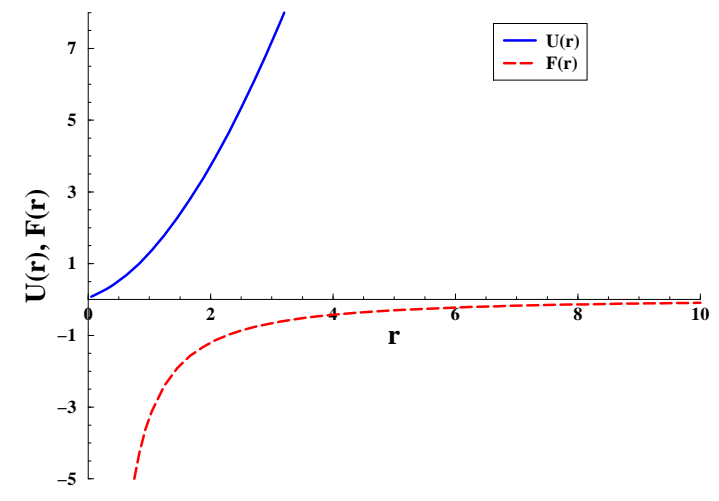

(a)

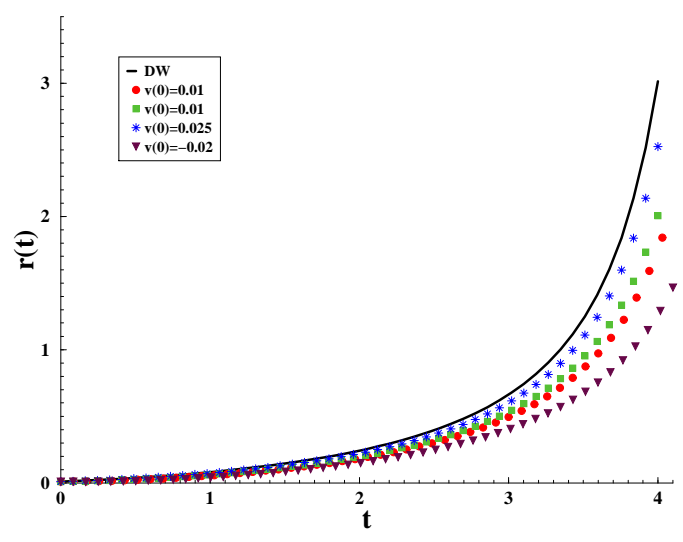

(b)

Figure 12: (a) $U(r)$ and $F(r)$ for type III solutions when $k=-1, M=-1 / 10$ and $b^{2}<\frac{1}{(D-2)}$, (b) Domain wall motion and geodesics for $V_{0}=-1, \hat{V}_{0}=1$, $\phi_{0}=1$ and $\beta=1 / \sqrt{6}$.

\subsection{4 $V_{0}<0, M<0, b^{2}<\frac{1}{(D-1)}$}

Here $U(r)$ is always positive while $F(r)$ is negative in the range shown in Fig.12. The domain wall and the geodesics diverge after some time near the end of the range where (49) has a solution.

\subsection{5 $V_{0}<0, M<0, \frac{1}{(D-1)}<b^{2}<1$}

In this case $U(r)$ is always positive while $F(r)$ is negative for a small range as seen in Fig.13. There are several shortcuts in the region where the domain wall equation of motion has solution; nevertheless, there is a threshold velocity after which the geodesics can not return.

\subsection{6 $V_{0}<0, M<0, b^{2}>1$}

Now $U(r)$ is always positive and $F(r)$ will determine the initial condition for the domain wall equation of motion. Results are in Fig.14. 


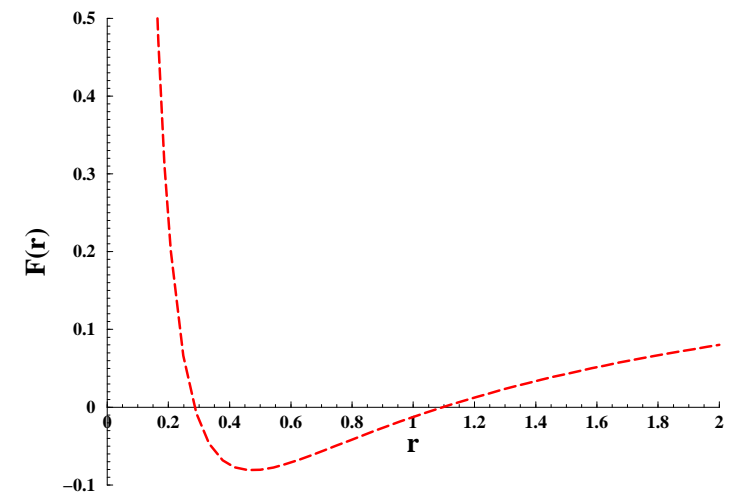

(a)

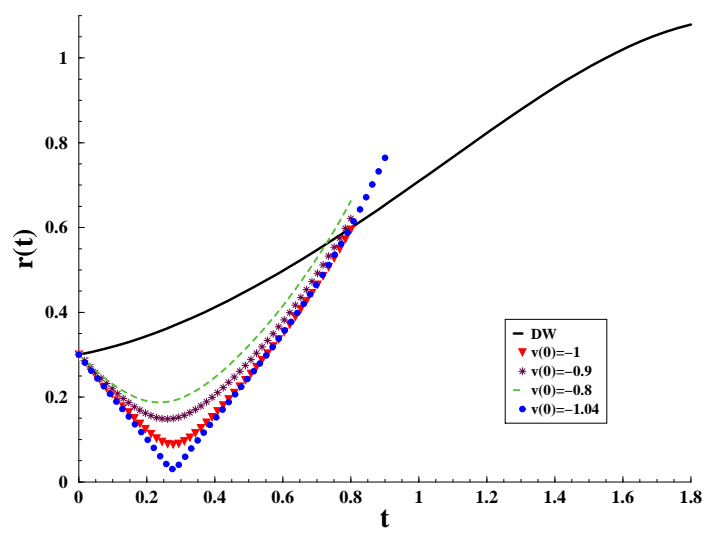

(b)

Figure 13: (a) $F(r)$ for type III solutions when $k=-1, M=-1 / 10$ and $\frac{1}{(D-2)}<b^{2}<1$, (b) Domain wall motion and geodesics for $V_{0}=-1, \hat{V}_{0}=1$, $\phi_{0}=1$ and $\beta=1 / \sqrt{2}$.

\subsection{Domain Wall Time and Time Delays}

The time delay between the photon traveling on the domain wall and the gravitons traveling in the bulk [23] can be calculated as follows. Since the signals cover the same distance,

$$
\int \frac{d \tau_{\gamma}}{r\left(\tau_{\gamma}\right)}=\int \frac{d t_{g}}{r_{g}\left(t_{g}\right)} \sqrt{U\left(r_{g}\right)-\frac{\dot{r}_{g}(t)^{2}}{U\left(r_{g}\right)}}
$$

the difference between photon and graviton time of flight can approximately be written as

$$
\frac{\Delta \tau}{r} \simeq \int_{0}^{\tau_{f}+\Delta \tau} \frac{d \tau_{\gamma}}{r\left(\tau_{\gamma}\right)}-\int_{0}^{\tau_{f}} \frac{d \tau_{g}}{r\left(\tau_{g}\right)}
$$

or in terms of the bulk time

$$
\Delta \tau \simeq r\left(t_{f}\right) \int_{0}^{t_{f}} d t\left(\frac{1}{r_{g}(t)} \sqrt{U\left(r_{g}\right)-\frac{\dot{r}_{g}(t)^{2}}{U\left(r_{g}\right)}}-\frac{1}{r(t)} \frac{d \tau}{d t}\right)
$$




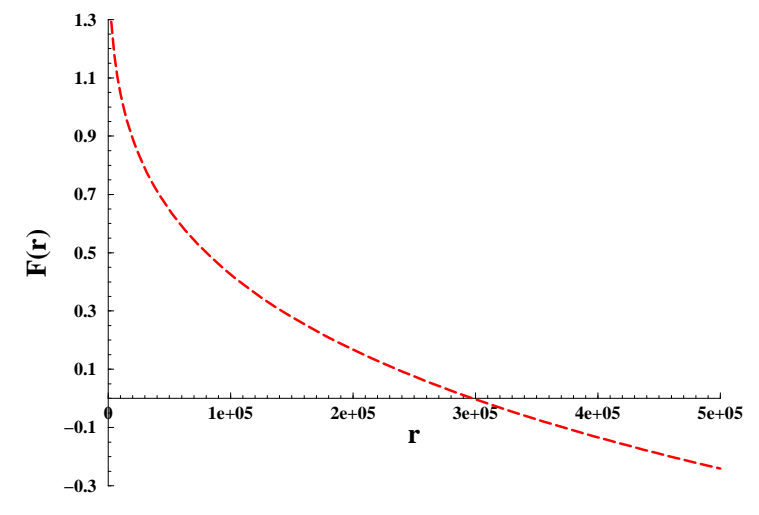

(a)

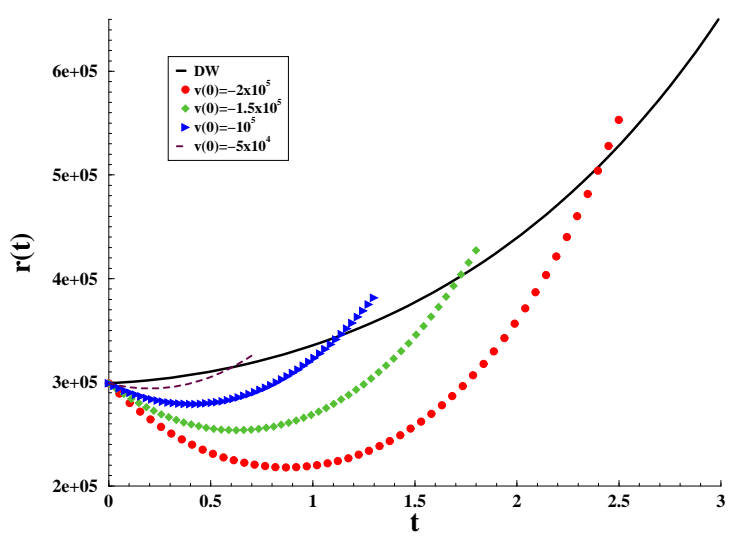

(b)

Figure 14: (a) $F(r)$ for type III solutions when $M=-1 / 10$ and $b^{2}>1$, (b) Domain wall motion and geodesics for $V_{0}=-1, \hat{V}_{0}=1, \phi_{0}=1$ and $\beta=\sqrt{5} / 2$.

The elapsed bulk time $t$, the corresponding domain wall time $\tau$ and the delays (83) can be computed.

\section{A Six-Dimensional Model}

We consider a six-dimensional model, such as the one constructed by Kanti et. al. [41]. We also search for a solution of six-dimensional Einstein equation in AdS space of the form

$$
d s^{2}=-n^{2}(t, y, z) d t^{2}+a^{2}(t, y, z) d \Sigma_{k}^{2}+b^{2}(t, y, z)\left\{d y^{2}+c^{2}(t, y, z) d z^{2}\right\}
$$

where $d \Sigma_{k}^{2}$ represents the metric of the three dimensional spatial sections with $k=-1,0,1$ corresponding to a hyperbolic, a flat and an elliptic space, respectively.

The total energy-momentum tensor can be decomposed in two parts corresponding to the bulk and the brane as

$$
\tilde{T}_{N}^{M}=\breve{T}_{N}^{M(B)}+T_{N}^{M(b)},
$$


where the brane contribution can be written as

$$
T_{N}^{M(b)}=\frac{\delta\left(z-z_{0}\right)}{b c} \operatorname{diag}(-\rho, p, p, p, \hat{p}, 0) .
$$

An analogous development of section 3.3 for this six dimensional model provides the following Darmois-Israel conditions

$$
\begin{aligned}
\frac{\left[\partial_{z} a\right]}{a_{0} b_{0} c_{0}} & =-\frac{\kappa_{(6)}^{2}}{4}(p-\hat{p}+\rho), \\
\frac{\left[\partial_{z} b\right]}{b_{0}^{2} c_{0}} & =-\frac{\kappa_{(6)}^{2}}{4}\{\rho-3(p-\hat{p})\}, \\
\frac{\left[\partial_{z} n\right]}{b_{0} c_{0} n_{0}} & =\frac{\kappa_{(6)}^{2}}{4}\{\hat{p}+3(p+\rho)\} .
\end{aligned}
$$

A metric of the form (84) satisfying six dimensional Einstein equations is given by

$$
d s^{2}=-h(z) d t^{2}+\frac{z^{2}}{l^{2}} d \Sigma_{k}^{2}+h^{-1}(z) d z^{2},
$$

where here

$$
d \Sigma_{k}^{2}=\frac{d r^{2}}{1-k r^{2}}+r^{2} d \Omega_{(2)}^{2}+\left(1-k r^{2}\right) d y^{2},
$$

and

$$
\begin{array}{cl}
h(z)=k+\frac{z^{2}}{l^{2}}-\frac{M}{z^{3}} & , \quad \text { for AdS-Schwarzschild bulk, } \\
h(z)=k+\frac{z^{2}}{l^{2}}-\frac{M}{z^{3}}+\frac{Q^{2}}{z^{6}} \quad, & \text { for AdS-Reissner-Nordström bulk, }
\end{array}
$$

with $l^{-2} \propto-\Lambda$ ( $\Lambda$ being the cosmological constant), which describes a black hole in the bulk, located at $z=0$.

Following [12], we find a further solution by means of a $Z_{2}$ symmetry inverting the space with respect to the brane position. That is, considering a metric of the form

$$
d s^{2}=-A^{2}(z) d t^{2}+B^{2}(z) d \Sigma_{(4)}^{2}+C^{2}(z) d z^{2}
$$

and the brane to be defined at $z=z_{0}$, there is a solution given by

$$
\begin{array}{cl}
A(z), B(z), C(z) & , \quad \text { for } \quad z \leq z_{0}, \\
A\left(z_{0}^{2} / z\right), B\left(z_{0}^{2} / z\right), C\left(z_{0}^{2} / z\right) \frac{z_{0}^{2}}{z^{2}} & , \quad \text { for } \quad z \geq z_{0} .
\end{array}
$$


The $Z_{2}$-symmetry corresponds to $z \rightarrow z_{0}^{2} / z$.

The static brane still has to obey the Darmois-Israel conditions (87), which for the metric (88) are written as

$$
\begin{aligned}
\frac{\left[\partial_{z} a\right]}{a_{0}^{2} c_{0}} & =-\frac{\kappa_{(6)}^{2}}{4} \rho, \\
\frac{\left[\partial_{z} n\right]}{a_{0} c_{0} n_{0}} & =\frac{\kappa_{(6)}^{2}}{4}(4 p+3 \rho),
\end{aligned}
$$

where here

$$
\left[\partial_{z} a\right]=-\frac{2}{l} \quad \text { and } \quad\left[\partial_{z} n\right]=-\frac{h^{\prime}\left(z_{0}\right)}{\sqrt{h\left(z_{0}\right)}}
$$

\subsection{The Shortest Cut Equation}

We consider a static version of the metric (84) with $k=0$

$$
d s^{2}=-n^{2}(z) d t^{2}+a^{2}(z) f^{2}(r) d r^{2}+b^{2}(z) d y^{2}+d^{2}(z) d z^{2},
$$

where the graviton path is defined equating (96) to zero. Therefore,

$\int_{r_{0}}^{r} f\left(r^{\prime}\right) d r^{\prime}=\int_{t_{0}}^{t} \frac{\sqrt{n^{2}(z)-b^{2}(z) \dot{y}^{2}-d^{2}(z) \dot{z}^{2}}}{a(z)} d t \equiv \int_{t_{0}}^{t} \mathcal{L}[y(t), \dot{y}(t), z(t), \dot{z}(t) ; t] d t$

which naturally defines a lagrangian density. The Euler-Lagrange equations of $\mathcal{L}$ define the graviton path. We first choose to work at a constant $y$ to check on the very possibility of (96) allowing shortcuts. In this case the resulting equation is simple but far from trivial,

$$
\ddot{z}+\left(\frac{a^{\prime}}{a}-2 \frac{n^{\prime}}{n}+\frac{d^{\prime}}{d}\right) \dot{z}^{2}+\left(\frac{n n^{\prime}}{d^{2}}-\frac{a^{\prime}}{a} \frac{n^{2}}{d^{2}}\right)=0 .
$$

Notice that this case is equivalent to consider the problem in five dimensions with the metric shown in [12].

The most general case includes a $y$ dependence on the graviton path; however, this dependence turns out to be superflous and does not affect the $z$-equation since (98) is independently satisfied. This conclusion is not surprising if we notice that the metric (96) is $y$-independent.

For $k \neq 0$ cases we can also consider (98) as the shortcut equation if we assume the existence of a $y$-symmetry in our problem. Our model represents a generalization of [12]. 


\subsection{AdS-Schwarzschild Bulk}

From the Darmois-Israel conditions (94) together with (95) we have

$$
\begin{aligned}
\frac{h}{z_{0}^{2}} & =\frac{\kappa_{(6)}^{4} \rho^{2}}{64}, \\
\frac{h^{\prime}}{2 z_{0}} & =-\frac{\kappa_{(6)}^{4} \rho^{2}}{64}(4 \omega+3),
\end{aligned}
$$

and we can obtain the black hole mass $M$ as a function of the brane energy density $\rho$, while $\rho$ is fixed by a fine-tunning,

$$
\begin{aligned}
\frac{M}{z_{0}^{5}} & =\frac{2}{5} \frac{k}{z_{0}^{2}}-(\omega+1) \frac{\kappa_{(6)}^{4} \rho^{2}}{40} \\
\frac{\kappa_{(6)}^{4} \rho^{2}}{64} & =-\frac{3 k}{z_{0}^{2}(8 \omega+3)}-\frac{5}{(8 \omega+3) l^{2}},
\end{aligned}
$$

where $\omega=p / \rho$.

As we saw in the previous section, the shortcuts in six dimensions are determined from (98).

If a shortcut exists, there must be a time $t=v$ in the graviton path when $\dot{z}(v)=0$ and $\ddot{z}(v) \geq 0$. Thus, (98) evaluated at this point will give

$$
\ddot{z}(v)+h\left(z_{v}\right)\left(\frac{h^{\prime}\left(z_{v}\right)}{2}-\frac{h\left(z_{v}\right)}{z_{v}}\right)=0 .
$$

It is obvious that this minimum must be between the brane and the event horizon $z_{h}$, if a horizon exists. Otherwise, there is no turning point in the path since the graviton can not return after it goes through the event horizon. Hence, $h\left(z_{v}\right)>0$. Thus, from (103) we require

$$
F\left(z_{v}\right)=\frac{h^{\prime}\left(z_{v}\right)}{2}-\frac{h\left(z_{v}\right)}{z_{v}} \leq 0 \quad \text { for } \quad z_{h}<z_{v}<z_{0} .
$$

Using (90) this implies

$$
F\left(z_{v}\right)=\frac{5}{2} \frac{M}{z_{v}^{4}}-\frac{k}{z_{v}} \leq 0 .
$$


This equation has a zero in $z=z_{f} \neq 0$ for $k \neq 0$

$$
z_{f}^{3}=\frac{5}{2 k} M
$$

For $k=0,-1$ there is no positive root. Since the mass is positive, $F(z)>0$ everywhere preventing the coexistence of shortcuts and horizons.

On the other hand, for $k=1$ there is one real and positive root, which must satisfy $z_{f}<z_{0}$ in order to have shortcuts. Taking into account (101) and the fact that $\varepsilon^{2}$ must be positive in (102), ${ }^{2}$ we have a first restriction for $\omega$,

$$
\omega+1>0 .
$$

The positivity of the black hole mass gives a second restriction for $\omega$,

$$
-1<\omega<-\frac{3}{4}
$$

and also for the brane position,

$$
\frac{z_{0}^{2}}{l^{2}}<-\frac{\omega+3 / 4}{\omega+1}
$$

If we follow both (107) and (108) together with the fine-tunning for the energy (102), we will have several shortcuts in AdS-Schwarzschild bulks with shielded singularity. In Figs. 15 and 16 we illustrate an example with $\omega=$ $-4 / 5, z_{0}=1 / 3$, and $l=1$. Notice in Fig. 15 that the horizon appears before the brane.

Since this case is equivalent to consider the problem in five dimensions with $h(z), M$ and $\rho$ given in [12], analogous results are obtained [11].

\subsection{AdS-Reissner-Nordström Bulk}

From the Darmois-Israel conditions (94) we have for the black hole mass and charge,

$$
\begin{aligned}
\frac{M}{z_{0}^{5}} & =\frac{2 k}{z_{0}^{2}}+\frac{8}{3 l^{2}}+\frac{\kappa_{(6)}^{4}}{24} \rho^{2} \omega \\
\frac{Q^{2}}{z_{0}^{8}} & =\frac{k}{z_{0}^{2}}+\frac{5}{3 l^{2}}+\frac{8 \omega+3}{3} \frac{\kappa_{(6)}^{4} \rho^{2}}{64} .
\end{aligned}
$$

\footnotetext{
${ }^{2}$ From now on, we will denote $\varepsilon^{2}=\kappa_{(6)}^{4} \rho^{2} / 64$ in six dimensions.
} 


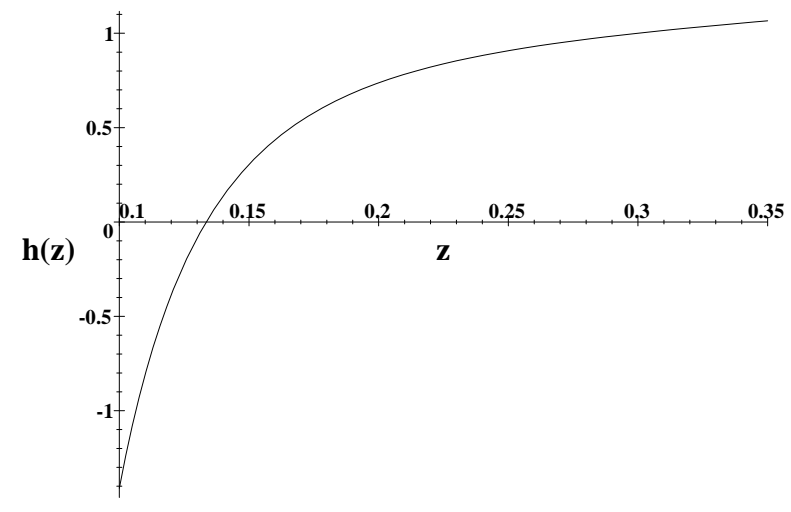

Figure 15: $h(z)$ in six-dimensional AdS-Schwarzschild bulk with the brane located at $z=1 / 3$. Notice that the singularity is shielded by a horizon.

At this stage it is convenient to carefully study the possibility of existence of shortcuts for every value of $k$.

As it was found in the AdS-Schwarzschild case, (104) determines the existence of shortcuts. Using (91) we see that (104) has a zero in $z=z_{f} \neq 0$ when

$$
\frac{5}{2} M z_{f}^{3}-4 Q^{2}-k z_{f}^{6}=0 .
$$

If $k=0$, we have a real root in

$$
z_{f}^{3}=\frac{8 Q}{5 M}
$$

If $k=1$, we have two roots in

$$
z_{f}^{3}=\frac{5}{4} M \pm \frac{1}{4} \sqrt{25 M^{2}-64 Q^{2}}
$$




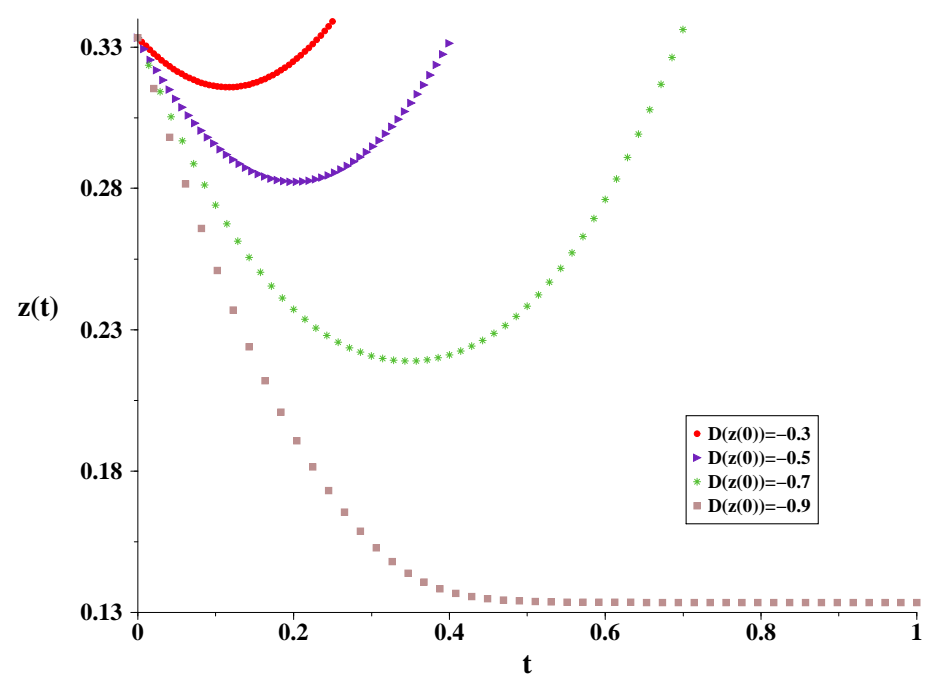

Figure 16: Shortcuts for several initial velocities in six-dimensional AdSSchwarzschild bulk. Notice that there is a threshold initial velocity for which the graviton can not return to the brane and falls into the event horizon.

Finally, if $k=-1$, we have

$$
z_{f}^{3}=-\frac{5}{4} M \pm \frac{1}{4} \sqrt{25 M^{2}+64 Q^{2}} .
$$

Notice that $F(z)$ has at most one real and positive zero if $k=0,-1$ and at most two positive zeros if $k=1$.

Analysing $h(z)$ and its derivatives we conclude that for positive mass there is just one zero for $h^{\prime}(z)$, and hence, at most two horizons for $h(z)$.

When there is one horizon, $h^{\prime}(z)$ is negative before it and positive after, crossing $h(z)$ at the very horizon. If there are two horizons, $h^{\prime}(z)$ vanishes at a point between Cauchy and event horizons, being negative before this point and positive after, while $h(z)$ is positive at all points except between both horizons. Taking into account both the sign and zeros of these functions, 
$h^{\prime}(z)$ crosses $h(z)$ between the Cauchy horizon and the point at which $h^{\prime}(z)$ vanishes.

Since $h^{\prime}(z) / 2$ has the same sign as $h^{\prime}(z)$ and vanishes at the same point, and in the same way $h(z) / z$ has the same sign of $h(z)$ and vanishes at the same points, we conclude that, existing horizons, $F(z)$ necessarily vanishes at some point $z=z_{c}$ such that $0<z_{c} \leq z_{h}$. However, as we pointed out before, for $k=0$ or $k=-1$ there is only one positive root of $F(z)$. As $F(z)<0$ for $z<z_{c}$, then $F(z)>0$ for $z>z_{c}$. Thus, because $z_{c} \leq z_{h}, F(z)>0$ for $z>z_{h}$ contrary to what was required in (104). This implies that there are no shortcuts with $k=0$ or $k=-1$ when horizons exist.

In five dimensions the proof is very similar and we arrive to the same conclusion.

In the case $k=1 F(z)$ has two real, positive and distinct roots,

$$
\begin{aligned}
r_{1}^{3} & =\frac{5}{4} M-\frac{1}{4} \sqrt{25 M^{2}-64 Q^{2}}, \\
r_{2}^{3} & =\frac{5}{4} M+\frac{1}{4} \sqrt{25 M^{2}-64 Q^{2}} .
\end{aligned}
$$

This is the only situation where the shortcuts can coexist with a shielded singularity. In fact, this situation necessarily requires the second root of $F(z)$ being at some point before the brane position $z_{0}$. This also implies $F\left(z_{0}\right)<0$.

In addition, we must have both $Q^{2}$ and $M$ positive.

Given the fact that we have horizons, if the brane is not between them or at a horizon position, $h\left(z_{0}\right)>0$. Furthermore, in order to guarantee that the brane is located after the event horizon, we also need $h^{\prime}\left(z_{0}\right)>0$.

From the previous discussion we will have one or two horizons if and only if $h\left(r_{1}\right) \leq 0$.

In summary, shortcuts in bulks with shielded singularities can occur only if $k=1$ and also if the following conditions are supplied,

1. $h\left(z_{0}\right)>0$ and $h^{\prime}\left(z_{0}\right)>0$ to have both horizons before the brane.

2. $F\left(z_{0}\right)<0$ and $r_{2}<z_{0}$ to have shortcuts with shielded singularity.

3. $Q^{2}>0$ and $M>0$, which assures the positivity of the black hole mass and the square of the charge. 
4. $h\left(r_{1}\right) \leq 0$ in order to have horizons.

As we have done for the Schwarzchild case, we can analyze each condition and impose certain restrictions on $\omega, \rho^{2}$, and $z_{0}$. In short, by purely analytic considerations we conclude that shortcuts in bulks having no naked singularities and a static brane embedded in can only appear if $k=1$ and if the following conditions are satisfied, [11]

1. We must choose $\omega$ such that $-1<\omega<-3 / 4$;

2. Given $\omega$, the brane must be located at a position such that

$$
\frac{z_{0}}{l}<\frac{1}{2} \sqrt{-\frac{3+4 \omega}{1+\omega}}
$$

which is the same condition as AdS-Schwarzschild case (108);

3. Given (116), the energy $\varepsilon$ must satisfy

$\frac{1}{32 \omega^{2}}\left(-\frac{32 \omega z_{0}^{2}}{l^{2}}+9+3 \sqrt{-64 \omega \frac{z_{0}^{2}}{l^{2}}+9-64 \frac{z_{0}^{2}}{l^{2}} \omega^{2}}\right)<z_{0}^{2} \varepsilon^{2}<\frac{1}{8 \omega+3}\left(-3-\frac{5 z_{0}^{2}}{l^{2}}\right)$.

In this way, it turns out to be simple to find shortcuts in bulks with shielded singularities.

As an example, let us choose $\omega=-9 / 10$. From (116) we must have

$$
\frac{z_{0}}{l}<\frac{\sqrt{6}}{2}
$$

then we choose $l=1$ and $z_{0}=1$.

From (117) we have

$$
\frac{35}{24}+\frac{5}{72} \sqrt{41}<\varepsilon^{2}<\frac{40}{21},
$$

so we choose $\varepsilon=\sqrt{238 / 125}$.

In figure (17) we plot $h(z)$ with these conditions. Notice that the singularity is protected by an event horizon and the brane is at $z=z_{0}=1$.

In figure (18) we plot the graviton paths obtained from (98) under the previous conditions for a variety of initial velocities showing that, in fact, shortcuts appear when we choose the parameters following the complete analysis shown in this section.

The analysis in five dimensions can be performed analogously. 


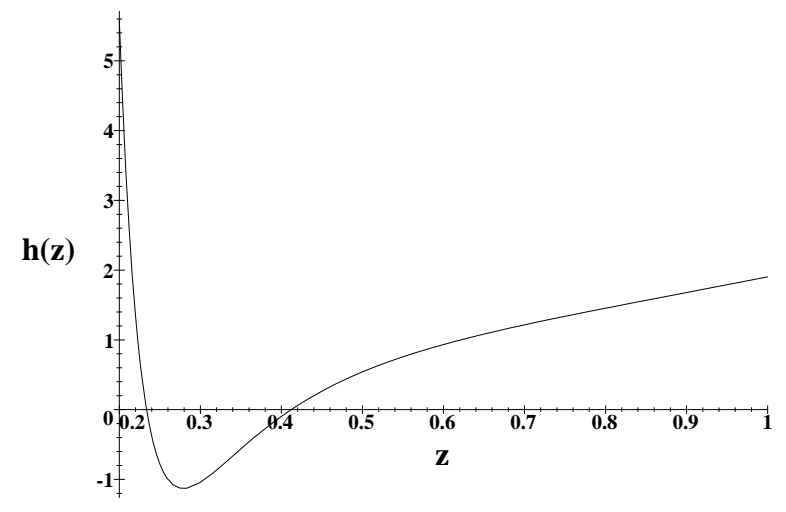

Figure 17: $h(z)$ in six-dimensional AdS-Reissner-Nordström bulk with the brane located at $z=1$. Notice that the singularity is shielded by two horizons.

\section{The FRW-Brane evolving in the Bulk}

The main reason to study the evolution of the brane from the point of view of the bulk is to simplify the analysis of gravitational signs leaving and subsequently returning to the brane. In fact in the static AdS backgound (51), the FRW brane (54) has a particularly simplified equation for a null geodesic in the bulk, $a=a(t)[11]$,

$$
\frac{\ddot{a}(t)}{a}+\frac{\dot{a}^{2}(t)}{a^{2}}\left(1-\frac{3 h^{\prime}(a) a}{2 h(a)}\right)+\frac{h(a)}{a}\left(\frac{h^{\prime}(a)}{2}-\frac{h}{a}\right)=0 .
$$

On the other hand, the evolution of the brane in the bulk, $a=a_{b}(t)$, at 


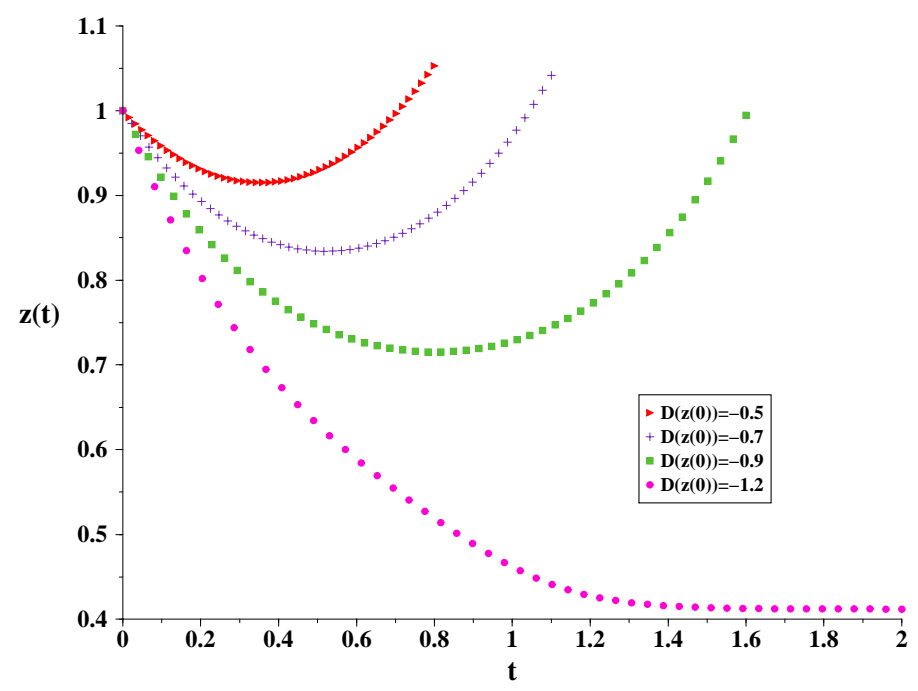

Figure 18: Shortcuts for several initial velocities in six-dimensional AdSReissner-Nordström bulk. Notice that there is threshold initial velocity for which the graviton can not return to the brane and falls into the event horizon.

early times, $a_{b}<<L_{c}$, is dictated by (66),

$$
\frac{d a_{b}}{d t}=\frac{h\left(a_{b}\right)}{\sqrt{1+\frac{h\left(a_{b}\right)}{\dot{a}_{b}(\tau)^{2}}}}=h\left(a_{b}\right)\left(1+\frac{k+\frac{a_{b}^{2}}{l^{2}}}{\frac{\Omega_{0}^{2}}{4\left(1+l^{2} \Lambda_{4}\right)} \frac{L_{c}^{2 q}}{l^{2} a_{b}^{2 q-2}}}\right)^{-1 / 2},
$$

where we used the fact that the quadratic term in the energy prevails. Thus,

$$
\frac{d a_{b}}{d t}=h\left(a_{b}\right)\left(1+\frac{4\left(1+l^{2} \Lambda_{4}\right)}{\Omega_{0}^{2}} \frac{a_{b}^{2 q}}{L_{c}^{2 q}}\left(1+k l^{2} /\left(a_{b}^{2}\right)\right)\right)^{-1 / 2},
$$

and since the observed cosmological constant is at most $\Lambda_{4} \sim H_{0}^{2}, \Lambda_{4} l^{2}<<1$ we have for $a_{b}<<L_{c}$,

$$
\frac{d a_{b}}{d t} \approx h\left(a_{b}\right)\left(1-\frac{2\left(1+l^{2} \Lambda_{4}\right)}{\Omega_{0}^{2}} \frac{a_{b}^{2 q}}{L_{c}^{2 q}}\left(1+k l^{2} /\left(a_{b}^{2}\right)\right)\right)
$$


Substituting the result for the evolution of the brane, $\dot{a}(t)=h(a)$, in the geodesic equation (118), we verify that it is satisfied. Therefore, the trajectory of the brane differs from the trajectory of the null geodesic by a term of the order $\left(\frac{a_{b}}{L_{c}}\right)^{2 q}$.

This means that for $a_{b}<<L_{c}$, the trajectory of the brane in the bulk is governed by

$$
\frac{d a_{b}(t)}{d t}=k+\frac{a_{b}^{2}}{l^{2}}
$$

Thus, if $k=0$,

$$
a_{b}(t)=\frac{a_{b}(0) l^{2}}{l^{2}-a_{b}(0) t}
$$

and, if $k=-1$,

$$
a_{b}(t)=\frac{2 l\left(l+a_{b}(0)\right)}{e^{2 t / l}\left(l-a_{b}(0)\right)+l+a_{b}(0)}-l .
$$

In this last situation, if the Universe begins under the Randall-Sundrum scale, $a(0)<l$, it will recolapse to the singularity in a finite time,

$$
t=\frac{l}{2} \ln \left(\frac{l+a_{b}(0)}{l-a_{b}(0)}\right) .
$$

There is an event horizon when $a=l$ if $k=-1$.

In the case of an elliptic Universe,

$$
a_{b}(t)=l \tan \left(\frac{t}{l}+\tan ^{-1}\left(\frac{a_{b}(0)}{l}\right)\right) .
$$

Starting at the singularity $t_{0}=\tau_{0}=a_{b}\left(t_{0}\right)=0$, we have

$$
a_{b}(t)=l \tan \left(\frac{t}{l}\right)
$$

Note that the evolution of the brane in the bulk is linear near the initial singularity $(a(t) \sim t$ for $t<<l)$, diverging at the critical time $t_{c}=\frac{\pi}{2} l$. In fact, the behaviour of all solutions is similar near the critical time

$$
\begin{aligned}
t_{c} & =\frac{l^{2}}{a_{b}(0)}, \\
t_{c} & =\frac{l}{2} \ln \left(\frac{l+a_{b}(0)}{a_{b}(0)-l}\right), \\
t_{c} & =\frac{\pi}{2} l-l \tan ^{-1}\left(\frac{a_{b}(0)}{l}\right),
\end{aligned}
$$


for $k=0,-1,+1$ respectively.

As we approach the critical time, $a_{b}(t)$ increases quickly. When $a_{b}(t) \sim$ $L_{c}$, equation (120) is no longer valid, and the trajectory of the brane is no longer a geodesic. Thus, for a very short period, from the point of view of the bulk the brane undergoes a phase transition. Before the critical time, from the point of view of the bulk, there is no time left for the remaining graviton geodesics to reach the brane.

For later times the evolution of the brane is softer, and shortcuts should appear. In fact, the numerical solutions of the brane evolution equation (66) and the null geodesics equation (118) in the bulk indicate the presence of shortcuts in late times universes, as exhibited in Fig. 19.

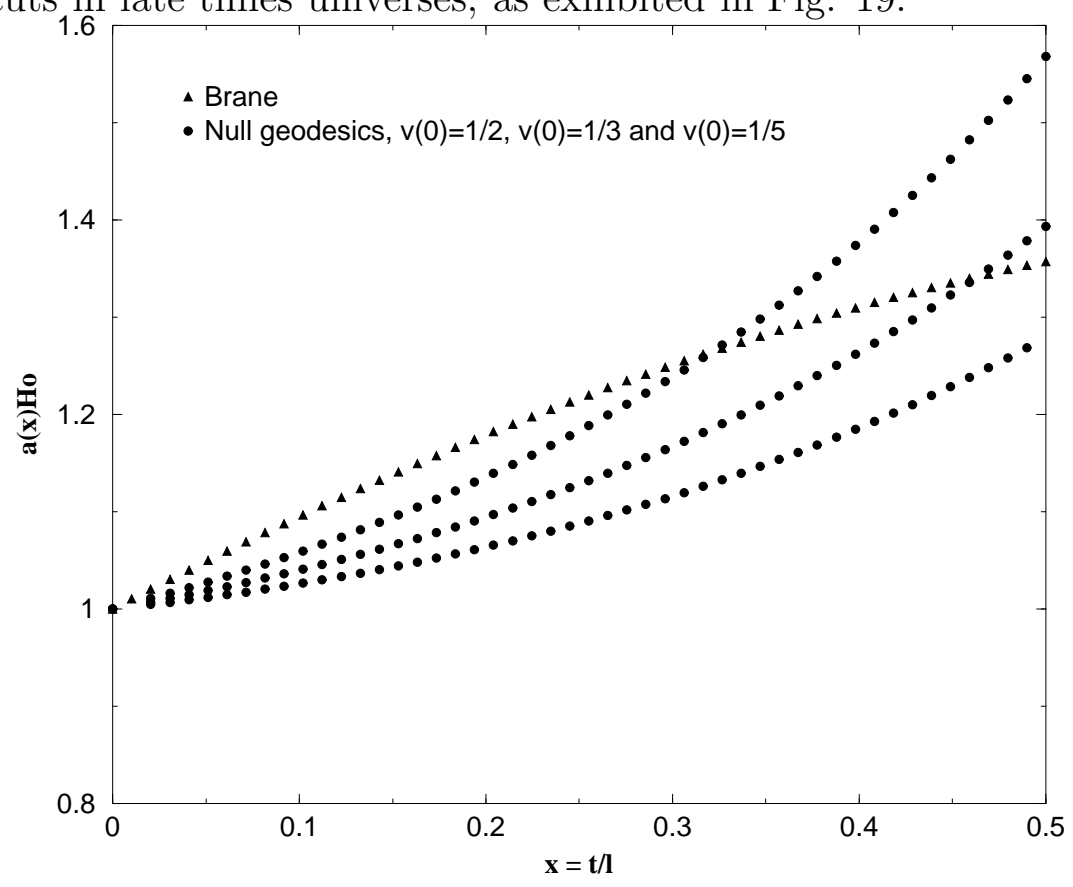

Figure 19: The trajectory of the brane in the bulk for $\Omega_{0}=2$, matter dominated era (MDE) with $k=1$. Also null geodesics starting in the brane with various initial velocities. 


\subsection{The Effect of Shortcuts in Late Times Universes}

The shortcuts just found for late times Universes could be used to probe the extra-dimensionality by the aparent violation of causality on the brane. Thus, suppose that in $\tau=t=0$ an object could emit eletromagnetic and gravitational waves and that we could be able to detect both signs in times $\tau_{\gamma}^{\prime}$ and $\tau_{g}^{\prime}$ respectively. We compute now the order of magnitude of the advance in time of the graviton. Since the signals cover the same distance on the brane,

$$
\int_{0}^{\tau_{\gamma}^{\prime}} \frac{d \tau_{\gamma}}{a_{b}\left(\tau_{\gamma}\right)}=\int_{0}^{t_{g}^{\prime}} \frac{d t_{g}}{a\left(t_{g}\right)} \sqrt{h(a)-\frac{\dot{a}\left(t_{g}\right)^{2}}{h(a)}} .
$$

Here $a$ denotes the coordinate defining the geodesic in the bulk, and differs from the coordinate of the brane $a_{b}$. In terms of the dimensionless parameters $y$ and $x, a=L y$ and $t=T x$ the last integral reads

$$
\int_{0}^{t_{g}^{\prime}} \frac{d t_{g}}{a\left(t_{g}\right)} \sqrt{h(a)-\frac{\dot{a}(t)^{2}}{h(a)}}=\int_{0}^{t_{g}^{\prime}} \frac{d t_{g}}{l} \sqrt{1+\frac{l^{2}}{L^{2} y^{2}}-\frac{l^{2}}{T^{2}} \frac{\dot{y}(x)^{2}}{y^{2}+y^{4} \frac{L^{2}}{l^{2}}}}
$$

Using the relation between the time on the brane and that in the bulk we express the above expression in terms of the time interval of the observer on the brane, and together with the Friedmann equations we get

$$
\begin{gathered}
\int_{0}^{\tau_{g}^{\prime}} \frac{d \tau_{g}}{l} \frac{1}{h\left(a_{b}\right)} \sqrt{\frac{y_{b}^{2} L^{2}}{l^{2}}+\Lambda_{4} L^{2} y_{b}^{2}+\frac{\Omega_{0}}{y_{b}^{q-2} \frac{L_{c}^{q}}{l^{2} L^{q-2}}} \sqrt{1+\frac{l^{2}}{L^{2} y^{2}}-\frac{l^{2}}{T^{2}} \frac{\dot{y}(x)^{2}}{y^{2}+y^{4} \frac{L^{2}}{l^{2}}}}} \\
=\int_{0}^{\tau_{g}^{\prime}} \frac{d \tau_{g}}{a_{b}\left(\tau_{g}\right)} \frac{1}{1+\frac{l^{2}}{L^{2} y_{b}^{2}}} \sqrt{1+l^{2} \Lambda_{4}+\frac{\Omega_{0} L_{c}^{q}}{y_{b}^{q}} \frac{L^{q}}{L^{q}}} \sqrt{1+\frac{l^{2}}{L^{2} y^{2}}-\frac{l^{2}}{T^{2}} \frac{\dot{y}(x)^{2}}{y^{2}+y^{4} \frac{L^{2}}{l^{2}}}} .
\end{gathered}
$$

If $L>>L_{c}>>l$, we obtain, at second order in $L_{c} / L$ and $l / L$,

$$
\begin{aligned}
\int_{0}^{\tau_{\gamma}^{\prime}} \frac{d \tau_{\gamma}}{a_{b}\left(\tau_{\gamma}\right)} & =\int_{0}^{\tau_{g}^{\prime}} \frac{d \tau_{g}}{a_{b}\left(\tau_{g}\right)}\left[1+\frac{1}{2} \frac{\Omega_{0}}{y_{b}^{q}} \frac{L_{c}^{q}}{L^{q}}+\frac{1}{2} l^{2} \Lambda_{4}-\frac{l^{2}}{L^{2}} \frac{1}{y_{b}^{2}}\right. \\
& \left.+\frac{1}{2} \frac{l^{2}}{L^{2} y^{2}}-\frac{1}{2} \frac{l^{4}}{T^{2} L^{2}} \frac{\dot{y}(x)^{2}}{y^{4}}\right] .
\end{aligned}
$$

Thus, at first order, the time difference between the photon and the graviton is corrected in the integrand by terms of order $\frac{L_{g}^{q}}{a_{b}^{q}}$. Today this factor is 
at most $10^{-58}$ and in the time of decoupling $10^{-46}$, showing that the time advance of the graviton can be safely neglected and is of no physical significance, in spite of the fact that the trajectory of the brane is distinctively different from the null geodesic.

\subsection{The Effect of Shortcuts in The Early Universe}

From the analysis developed so far we learned that the periods of evolution of the Universe differ by the scale that defines the physical significance of the shortcuts. When $a_{b}<<L_{c}$, the trajectory of the brane in the bulk differs from the extreme geodesics by $\left(a_{b} / L_{c}\right)^{2 q}$ and the shortcuts do not appear since the brane itself provides the graviton geodesic.

In the period when $a_{b}>>L_{c}$, the trajectory of the brane is far from a null geodesic and shortcuts appear, but they are not significant since the skin depth of the graviton in the bulk is defined by the parameter $l<<L_{c}<<a_{b}$. The difference between the time intervals of the photon and the graviton is of the order $\left(L_{c} / a_{b}\right)^{q}$.

However, from the continuity of the evolution of the brane in the bulk, we expect that there is also an intermediate situation $a_{b} \sim L_{c}$ when physically important shortcuts could appear since the evolution of the brane is far enough from a geodesic. Indeed, in Fig. 20, rescaling the geodesics in the bulk we can observe the behaviour of the brane as compared to the geodesics that start on it at a later time. It is clear that these shortcuts are serious mediators of homogenization of the matter on the brane in the era before nucleosynthesis [13], [14].

From the evolution of the brane in the bulk in the intermediate epoch we thus conclude that there is a critical age $t_{c}$, after which the gravitational waves leaving the brane return before the arrival of the photons released at the same time as the gravitons. The behaviour of the geodesic in the bulk shows that any geodesic starting on the brane at a certain instant will be singular at a time later than the critical time, indicating that it will return to the brane. Thus, information leaks between regions which apparently are causally disconnected at times $t>t_{c}$.

In order to study the horizon problem we now compare, at a certain time $\tau_{n}$ previous to nucleosynthesis, the graviton horizon $R_{g}$ with the observable proper distance of the universe (from the radiation decoupling until today) 


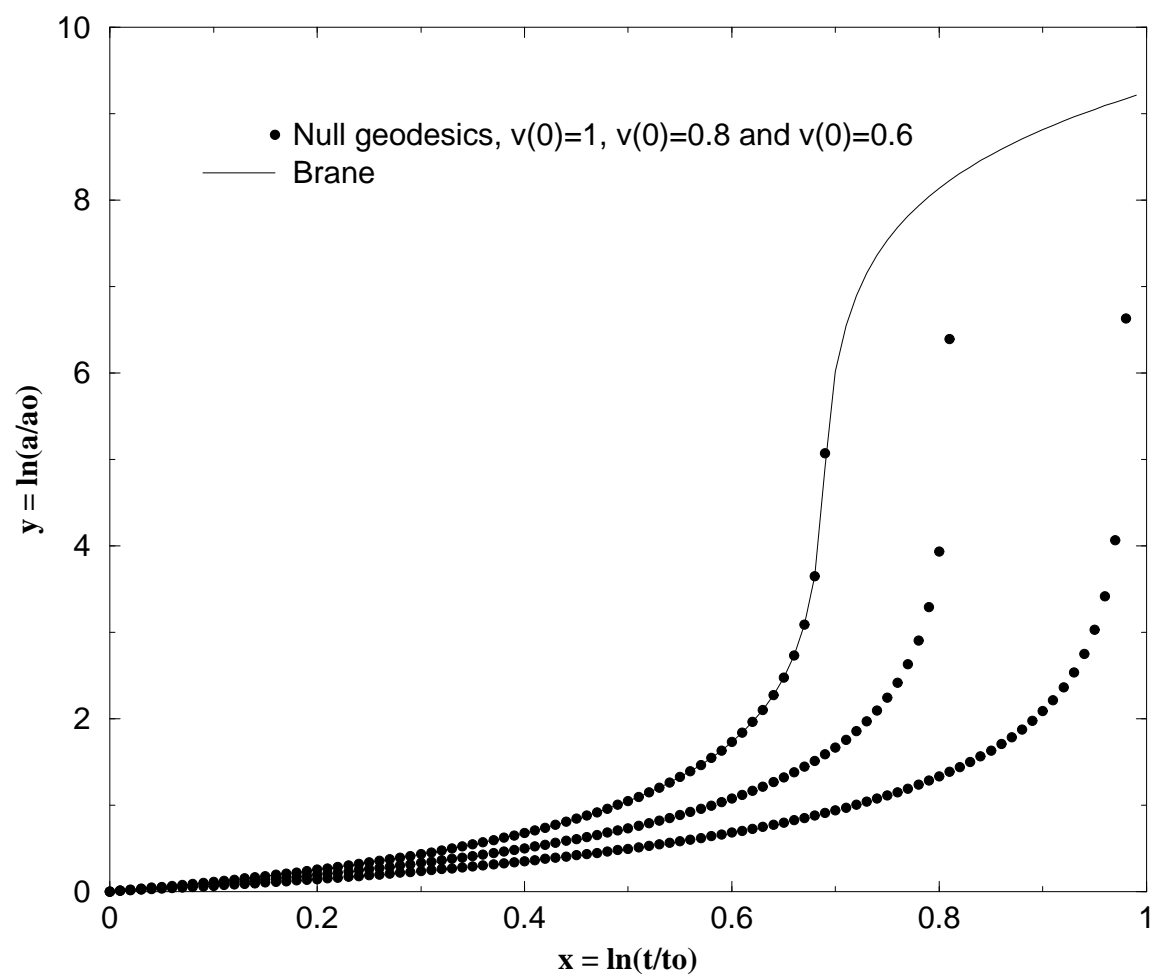

Figure 20: The trajectory of the brane in the bulk leaving $a=0.01 L_{c}$ in the RDE. Geodesics in the bulk with different initial velocities are exhibited intercepting the brane after the critical time $t_{c}$.

[15]. Since the graviton evolves in a bulk geodesic,

$$
\begin{aligned}
R_{g} \equiv & \int \frac{d r}{\sqrt{1-k r^{2}}}=\int \frac{d t}{a} \sqrt{h(a)-\frac{\dot{a}^{2}}{h(a)}}=\int \frac{d \tau}{a} \frac{\sqrt{h\left(a_{b}\right)+\dot{a}_{b}^{2}}}{h\left(a_{b}\right)} \sqrt{h(a)-\frac{\dot{a}^{2}}{h(a)}} \\
= & \int_{0}^{\tau_{n}} \frac{d \tau}{a_{b}} \frac{1}{1+l^{2} / a_{b}^{2}} \sqrt{1+\Lambda_{4} l^{2}+\frac{\Omega_{0} L_{c}^{q}}{a_{b}^{q}}\left(1+\frac{\Omega_{0} L_{c}^{q}}{4 a_{b}^{q}\left(1+\Lambda_{4} l^{2}\right)}\right) \times} \\
& \times \sqrt{1+\frac{l^{2}}{L^{2} y^{2}}-\frac{l^{2}}{T^{2}} \frac{\dot{y}(x)^{2}}{y^{2}+y^{4} \frac{L^{2}}{l^{2}}}},
\end{aligned}
$$


while on the brane, the size of the observable Universe is $R=\int \frac{d \tau}{a_{b}(\tau)}$.

We use the known results for the usual particle horizon

$$
R=\frac{1}{\sqrt{\Omega_{0}} H_{0} a_{b 0}} \int_{z(\tau)}^{z(0)} z^{-q / 2} d z=\frac{2}{(q-2) \sqrt{\Omega_{0}} H_{0} a_{b 0}}\left(z(\tau)^{1-q / 2}-z(0)^{1-q / 2}\right)
$$

where $z(\tau)=a_{b 0} / a_{b}(\tau)$. Today,

$$
R \sim \frac{2}{\sqrt{\Omega_{0}} H_{0} a_{b 0}} \equiv R_{0}
$$

For the computation of the graviton horizon we work in a primordial era before nucleosynthesis. Thus, in the Friedmann equation we can neglect the usual cosmological term as well as the curvature term. At an epoch between Planck era and nucleosynthesis, $l<<a_{b}<<H_{0}$, it is safe to neglect terms involving $l^{2} / L^{2}$ in (128), and we find

$$
R_{g} \approx \int_{0}^{\tau_{n}} \frac{d \tau}{a_{b}} \sqrt{1+\frac{\Omega_{0} L_{c}^{q}}{a_{b}^{q}}+\frac{\Omega_{0}^{2} L_{c}^{2 q}}{4 a_{b}^{2 q}}} .
$$

Using the Friedmann equation we get

$$
R_{g}=\frac{1}{\Omega_{0}^{1 / 2} H_{0} a_{b 0}} \int_{z\left(\tau_{n}\right)}^{z(0)} \frac{d z}{z^{2}} \frac{\left(1+\frac{\Omega_{0} H_{0}^{2} l^{2}}{2} z^{4}\right)}{\sqrt{1+\frac{\Omega_{0} H_{0}^{2} l^{2}}{4} z^{4}}} .
$$

The integral diverges for arbitrarly high redshifts, proving that the horizon problem is potentially solvable. The behaviour of this integral can be determined,

$$
R_{g} \approx \frac{l}{a_{b 0}} z(0)
$$

Comparing with the size of the Universe today we have

$$
\frac{R_{g}}{R_{0}} \sim \frac{\sqrt{\Omega_{0}}}{2} H_{0} l z(0)
$$

It looks like shortcuts are not enough to solve the horizon problem since we would need to go back in time to $z(0) \sim\left(H_{0} l\right)^{-1} \sim 10^{29}, 10^{11}$ times higher than the redshift at the Planck time on the brane associated with the fundamental scale of gravity, $\kappa_{5}$. 
We may note, however, that there are actually two related time scales. In the primordial Universe the brane is evolving as a part of the bulk, with velocities close to that of light, and time intervals on the brane correspond to much longer intervals in the bulk. In fact, the relation between these scales is obtained from $t_{0}=\tau_{0}=a_{b}\left(\tau_{0}\right)=0$ and

$$
t=\int_{0}^{\tau} d \tau \frac{\sqrt{h\left(a_{b}\right)+\dot{a}_{b}^{2}(\tau)}}{h\left(a_{b}\right)} .
$$

For $a_{b}<<L_{c}$ we have $\tau<<l$, and one finds as a consequence

$$
t \approx \int_{0}^{\tau} d \tau \frac{\dot{a}_{b}(\tau)}{h\left(a_{b}\right)}=\int_{0}^{a_{b}(\tau)} \frac{d a_{b}}{k+a_{b}^{2} / l^{2}}
$$

This implies for the example of a closed universe with $\Omega_{0}=2$,

$$
t=l \arctan \left(\frac{a_{b}(\tau)}{l}\right) .
$$

Therefore, when $a_{b}(\tau) \sim l$, that is, when the brane time is $\tau \sim 10^{-67} s$, the corresponding bulk time $t \sim \frac{\pi}{4} l \sim 10^{-11}$ s is much larger than the Planck scale, $t>>M_{(5)}^{-1}$.

If we assume that quantization is mandatory according to the bulk Planck energy scale, geodesics that start in the bulk with $z(0) \sim\left(H_{0} l\right)^{-1}$ should be sufficient to homogenize the Universe before nucleosynthesis reaching $R_{g} \sim 1$. In order to verify this, let us note that we can study analytically the whole causal structure of the gravitational signs for a $k=0$ Universe.

The geodesic equation, (118), is quite simple for purely AdS $k=0$ spacetimes

$$
\frac{1}{a^{2}} \frac{d a}{d t}=\frac{v(0)}{a(0)^{2}}
$$

Thus, a geodesic that starts on the brane at $a=a(0)$ and $t=0$, with initial velocity $v(0)$, returns to it when

$$
t_{r} \approx \frac{a(0)}{v(0)} .
$$


The expression for the gravitational horizon can also be integrated

$$
\begin{aligned}
R_{g} & =\int_{0}^{t_{r}} \frac{d t}{a} \sqrt{h(a)-\frac{\dot{a}^{2}}{h(a)}}=\int_{0}^{t_{r}} \frac{d t}{l} \sqrt{1-\frac{l^{4} \dot{a}^{2}}{a^{4}}} \\
& =\frac{t_{r}}{l} \sqrt{1-\frac{v(0)^{2} l^{4}}{a(0)^{4}}} .
\end{aligned}
$$

Using the relation between the returning time and the initial velocity,

$$
R_{g}=\frac{t_{r}}{l} \sqrt{1-\frac{l^{4}}{a(0)^{2} t_{r}^{2}}} .
$$

In order to relate the returning time to the redshift, we must integrate the relation between time of the bulk and time of the brane (66). We already know that from $a(0)$ to the critical period of transition a time of $t_{c} \sim \frac{l^{2}}{a(0)}$ has passed. After that, the evolution is dominated by the usual term in Friedmann equation and we can use

$$
\begin{aligned}
t_{r} & \approx \frac{l^{2}}{a(0)}+\int_{L_{c}}^{a_{r}} l \frac{d a_{b}}{a_{b}^{2} H} \approx \frac{l^{2}}{a(0)}+\int_{L_{c}}^{a_{r}} d a_{b} \frac{l}{\sqrt{\Omega_{0}} H_{0}} a_{b}^{q / 2-2} \\
& \approx \frac{l^{2}}{a(0)}+\frac{2 l}{(q-2) \sqrt{\Omega_{0}} H_{0} a_{b 0}}\left(z_{r}^{-q / 2+1}-10^{-15}\right),
\end{aligned}
$$

where $z_{r}$ must, of course, be greater than the redshift in the transition, $z_{L c} \sim 10^{15}$.

Substituting back in the expression of the gravitational horizon,

$$
\begin{aligned}
R_{g} & =\left[\frac{l}{a(0)}+\frac{2}{(q-2) \sqrt{\Omega_{0}} H_{0} a_{b 0}}\left(z_{r}^{-q / 2+1}-10^{-15}\right)\right] \\
& \times \sqrt{1-\left[1+\frac{2 a(0)}{(q-2) \sqrt{\Omega_{0}} l H_{0} a_{b 0}}\left(z_{r}^{-q / 2+1}-10^{-15}\right)\right]^{-2}} .
\end{aligned}
$$

When considering the interesting situation of a high initial redshift $z(0)=$ $a_{b 0} / a(0)>\left(H_{0} l\right)^{-1}$, this expression can be approximated by

$$
R_{g} \approx \frac{l}{a(0)} \sqrt{\frac{4 a(0)}{(q-2) \sqrt{\Omega_{0}} l H_{0} a_{b 0}}\left(z_{r}^{-q / 2+1}-10^{-15}\right)} .
$$


Comparing with the size of the horizon today we find

$$
\left(\frac{R_{g}}{R_{0}}\right)_{z_{r}} \approx \sqrt{\sqrt{\Omega_{0}} \frac{l H_{0}}{(q-2)}} \sqrt{z(0)\left(z_{r}^{-q / 2+1}-10^{-15}\right)} .
$$

Thus, as we have previously noted, if sufficiently large redshifts were available, the graviton horizon in a past epoch could be larger than the present size of the observable Universe.

We argue, however, that those high redshifts could be available. Indeed, if inflation takes place on the brane, high redshifts could be present in the beginning of the inflationary epoch.

Denoting the redshift when inflation ends by $z_{e}$, if the size of the present Universe, $R_{0}$, is expected to be in causal contact during inflation, we must reach at least a redshift in the beginning of inflation, $z(0)$, that solves

$$
\frac{a_{b 0} R_{0}}{z(0)}=H^{-1}\left(z_{e}\right)
$$

The unusual results in brane-world cosmology are expected if inflation ends before the transition time, when the quadratic term in Friedmann equation dominates. In this case we get

$$
\frac{a_{b 0} R_{0}}{z(0)}=\frac{1}{\Omega_{0} H_{0}^{2} l z_{e}^{4}} \quad \text { and } \quad z(0)=2 \sqrt{\Omega_{0}} H_{0} l z_{e}^{4}
$$

If $H\left(z_{e}\right) l>>1$, it is simple to note, from equation (66), that the evolution of the brane in the bulk is not altered during inflation. Thus, we can substitute the result(137) in the complete expression for the causal gravitational horizon (136),

$$
\left(\frac{R_{g}}{R_{0}}\right)_{z_{r}} \approx \sqrt{\frac{2 \Omega_{0}}{(q-2)}} H_{0} l z_{e}^{2} \sqrt{\left(z_{r}^{-q / 2+1}-10^{-15}\right)} .
$$

This equation tells us that in the time of nucleosyntesis, when $z_{r} \sim 10^{10}$, $R_{g} / R_{0} \sim 1$ for a model with inflation ending just before what would be the Planck era with $z_{e} \sim 10^{17}$ (where $z_{P l} \sim 10^{18}$ ). This proves that successfully inflationary models ending before the transition time necessarily make relevant changes in the causal structure of the universe. 
We are able to sketch the gravitational horizon for this kind of configuration. In Fig. 21 we show the behaviour of the Hubble horizon in comoving coordinates $H^{-1} a_{b}^{-1} / R_{0}$ and the graviton horizon $R_{g} / R_{0}$ for an inflationary model ending just before the Planck era, $z_{e}=10^{17}$ and producing the necessary number of e-folds to solve the horizon problem. The fraction of the Universe in causal contact by gravitational signs in the nucleosynthesis epoch is just the present horizon $R_{0}$. Today, the gravitational horizon would be $10^{5} R_{0}$.

\section{Conclusions}

We have shown that gravitational shortcuts in three braneworld models are common and there are many consequences.

In the brane wall model, where the Universe is replaced by a domain wall, we have proved that shortcuts may exist and above all abundant, which is a necessary condition in order to solve the homogeneity problem. The model shows interesting results as the delay of the time of flight inside the brane that can be comparable with the time of flight of the graviton itself. This lends further support for a thermalization via graviton exchange through the extra dimensions.

In the brane static models, the AdS-Schwarzschild and AdS-ReissnerNordström bulks also open up the possibility of having shortcuts provided both the spatial section has positive curvature and a set of strong restrictions on the brane intrinsic tension must be satisfied. Moreover, its location in the bulk has to be respected. It is interesting to notice that despite the fact that the charge contributes to have a negative $F\left(z_{0}\right)$ and thus facilitates the existence of shortcuts, there are more restrictive conditions for the energy coming from $Q^{2}>0$ and from the horizons equation which do not appear in the uncharged case. In this way, the results favor the existence of shortcuts in bulks with shielded singularities with the same conditions for $\omega$ and $z_{0}$ as the AdS-Schwarzschild case and also impose what is basically a fine-tunning in the energy that already exists in the uncharged model directly from the junction conditions.

Finally, studying the shortcut problem in the cosmological braneworld model from the point of view of the bulk, we have explicitly shown that shortcuts are indeed common in late time Universes, though they are ex- 


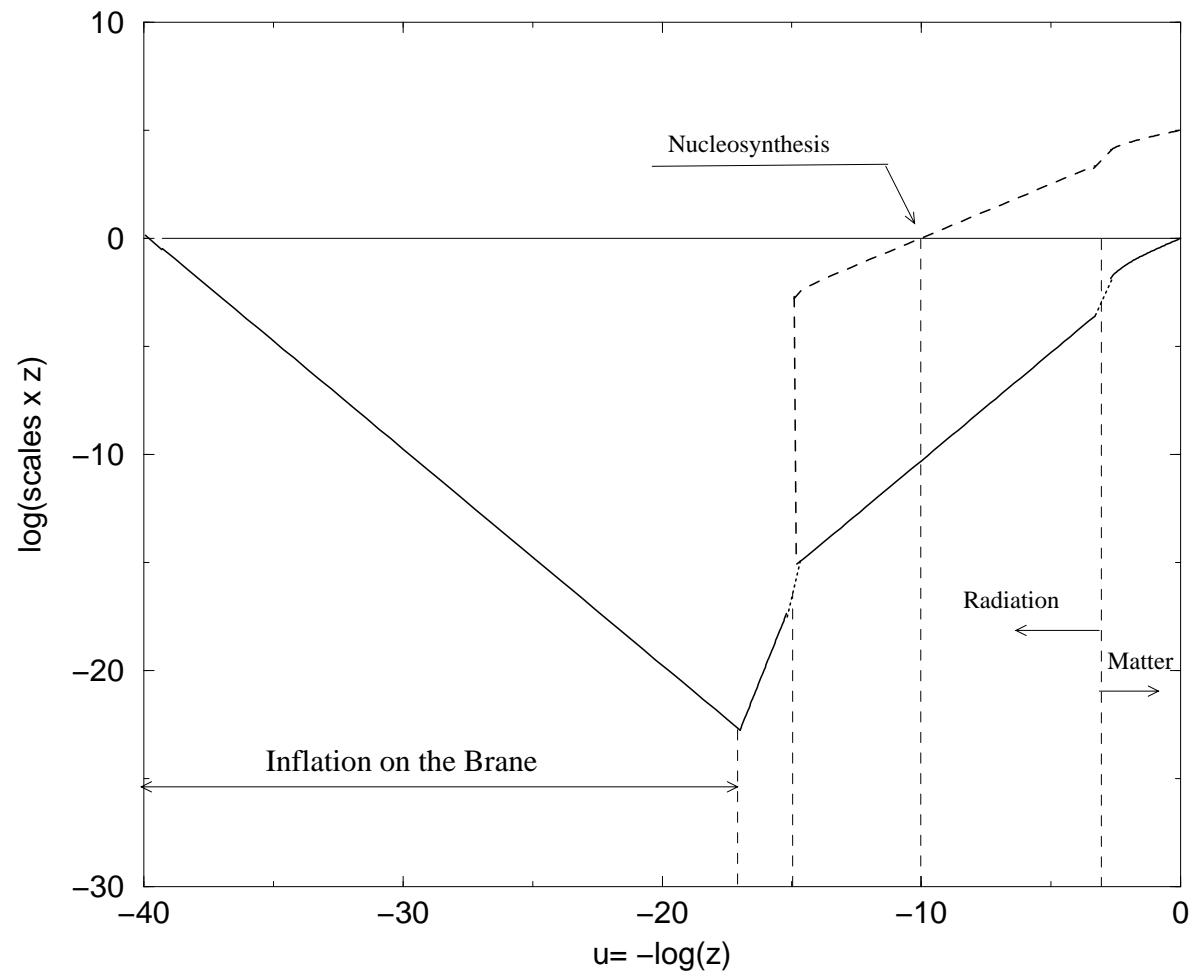

Figure 21: We plot in log-log scale the evolution of the fraction of graviton horizon and the present observable size, $R_{g} / R_{0}$ (dashed line) and the same for the Hubble horizon $a_{b}^{-1} H^{-1} / R_{0}$ (solid line), for an inflationary model in the brane that ends after Planck time $\left(u_{P L}=-\log \left(z_{P L}\right)=-18\right)$, and before transition time $\left(u_{T R}=-15\right)$, in $u_{\text {end }}=-\log \left(z_{\text {end }}\right)=-17$. The present scale would be under the de-Sitter horizon if redshifts like $u(0)=-39$ were available. This imply in strong modifications of the causal structure for gravitational signs after the transition time.

tremelly small and the time advance of the graviton can be safely neglected. However, we have also learned that gravitational signs may leave and subsequently return to the brane even in early universes. We have shown that those shortcuts exist and that the new scale of the model, $l$, implies in a minimum time scale for the reception of those signs by an observer on the 
brane. Before that critical time the brane itself evolves like a null-geodesic in the bulk. If high initial redshifts were available the shortcuts just found could solve the horizon problem without inflation. More important however, may be the effect of those shortcuts in an inflationary epoch on the brane.

Braneworld models incorporate two changes in the cosmology, namely, the modified Friedmann equation and the possibility of leaking of gravity in the extra dimension. Using the first of those modifications it was shown that, remarkably, the consistency equation is mantained in the brane-world formalism when the inflation is guided by a scalar field minimally coupled on the brane [20]. This consists in bad news for those who expect that brane cosmological configurations could probe the extra dimensionality of our Universe.

However, we have shown that with an inflationary epoch in the brane evolution the causal structure of the universe could be strongly modified. This could be a sign of an unusual evolution of the perturbations from the time they cross the de Sitter horizon, $H^{-1}$, during inflation, through the time they became causally connected again. In this case, there could be distinct predictions for the microwave background radiation structure even with the same consistency equation during inflation. Thus, further investigation on the dynamics of perturbations in inflationary brane-world models may prove useful to probe the dimensionality of space-time.

Acknowledgements: This work has been supported by Fundação de Amparo à Pesquisa do Estado de São Paulo (FAPESP) and Conselho Nacional de Desenvolvimento Científico e Tecnológico (CNPq), Brazil.

\section{References}

[1] M. Gasperini, G. Veneziano, The pre big-bang scenario in String Cosmology, CERN-TH (2002) 104; [hep-th/0207130].

[2] J. Polchinski, Superstring Theory vols. 1 and 2, Cambridge University Press, 1998.

[3] Gerard 't Hooft and M.J.G. Veltman, Annales Poincare Phys. Theor. A20 (1974) 69-94.

[4] P. van Nieuwenhuizen Phys. Rept. 68 (1981) 189-398. 
[5] M. Green, J, Schwarz and E. Witten, Superstring Theory, Vol. 1 and 2, Cambridge University Press, Cambridge, 1986.

[6] E. Witten Nucl. Phys. B433 (1995) 85.

[7] P. Horava and E. Witten Nucl. Phys. B460 (1996) 506.

[8] T. Kaluza, Sitzungsberichte Preussische Akademie der Wissenschaften K1 (1921) 966; O. Klein, Z. F. Physik 37 (1926) 895; O. Klein, Nature 118 (1926) 516.

[9] N. Arkani-Hamed, S. Dimopoulos and G. Dvali, Phys. Lett. B429 (1998) 263; I. Antoniadis, N. Arkani-Hamed, S. Dimopoulos and G. Dvali, Phys. Lett. B436 (1998) 257.

[10] P. Horava and E. Witten, Nucl. Phys.B475, 94 (1996).

[11] E. Abdalla, A. Casali, B. Cuadros-Melgar, Nucl. Phys. B644 (2002) 201; [hep-th/0205203].

[12] C. Csáki, J.Erlich, C. Grojean, Nucl. Phys. B604 (2001) 312; [hepth/0012143].

[13] H. Ishihara, Phys. Rev. Lett. 86 (2001) 381.

[14] R. Caldwell and D. Langlois, Phys. Lett. B511 (2001) 129; [grqc/0103070].

[15] D. J. Chung and K. Freese, Phys. Rev. D62 (2000) 063513; [hepph/9910235]. Phys. Rev. D61 (2000) 023511; [hep-ph/9906542].

[16] C. Csáki, M.Graesser, L. Randall and J. Terning, Phys. Rev. D62, (2000) 045015, [hep-th/9911406]; P. Binetruy, C. Deffayet, D. Langlois Nucl.Phys. B615, (2001) 219, [hep-th/0101234].

[17] E. Abdalla, B. Cuadros-Melgar, S. Feng, B. Wang, Phys. Rev. D65 (2002) 083512; [hep-th/0109024].

[18] Shin'ichi Nojiri, Sergei D. Odintsov, Akio Sugamoto, Mod. Phys. Lett. A17 (2002) 1269; [hep-th/0204065]. Shin'ichi Nojiri, Sergei D. Odintsov, JHEP 0112 (2001) 033; [hep-th/0107134]. Bin Wang, Elcio Abdalla, Ru-Keng Su, Mod. Phys. Lett. A17 (2002) 23; [hep-th/0106086]. 
[19] G. Giudice, E. Kolb, J. Lesgourgues and A. Riotto, CERN-TH (2002) 149; [hep-ph/0207145].

[20] G. Huey and J. Lidsey, Phys. Lett. B514, 217 (2001), [astroph/0104006]; A. Liddle and A. Taylor, Phys. Rev. D65, 041301 (2002), [astro-ph/0109412].

[21] G. Starkman, D. Stojkovic and M. Trodden, Phys. Rev. Lett. 87 (2001) 231303; Phys. Rev. D63 (2001) 103511.

[22] D. J. Chung and K. Freese; [astro-ph/0202066].

[23] E. Abdalla and A. G. Casali; [hep-th/0208008].

[24] G. Dvali and S. H. Tye Phys. Lett. B450 (1999) 72, [hep-th/9812483].

[25] F. Quevedo Class. Quant. Grav. 19 (2002) 5721-5779.

[26] S. Kashru, R. Kallosh, A. Linde, J. Maldacena, L. McAllister and S. Trivedi, [hep-th/0308055].

[27] J. W. Moffat; [hep-th/0208122].

[28] C. Lanczos, Phys. Zeits. 23 (1922) 539; Ann. der Phys. 74 (1924) 518.

[29] W. Israel, Nuovo Cimento 44B (1966) 1. Erratum: 48B (1967) 2.

[30] G. Darmois, Mem. Sciences Math. XXV (1927) ch. V.

[31] H.A. Chamblin and H.S. Reall, Nucl. Phys. B562 (1999) 133.

[32] Gibbons and S. Hawking, Phys. Rev. D15 (1977) 2738.

[33] P. Binétruy, C. Deffayet and D. Langlois, Nucl. Phys. B565 (2000) 269.

[34] P. Binétruy, C. Deffayet, U. Ellwanger and D. Langlois, Phys. Lett. B477 (2000) 285.

[35] D. Ida, JHEP 0009 (2000) 014; [gr-qc/9912002].

[36] L. Randall and R. Sundrum, Phys. Rev. Lett. 83, (1999) 3370, [hepth/9905221]; Phys. Rev. Lett. 83, (1999) 4690, [hep-th/9906064]. 
[37] P. Kraus, JHEP 9912 (1999) 011; [hep-th/9910149].

[38] P. Bowcock, C. Charmousis and R. Gregory, Class. Quant. Grav., 17 (2000) 4745 ; [hep-th/0007177].

[39] C. Csáki, M. Graesser, C. Kolda and J. Terning, Phys. Lett. B462 (1999) 34; [hep-ph/9906513].

[40] J. Cline, C. Grosjean and G. Servant, Phys. Rev. Lett. 83 (1999) 4245; [hep-ph/9906523].

[41] P. Kanti, R. Madden and K. A. Olive, Phys. Rev. D64 (2001) 044021; [hep-th/0104177]. 